\title{
Integrable $(2+1)$-dimensional systems of hydrodynamic type
}

\author{
A.V. Odesskii ${ }^{1}$, V.V. Sokolov ${ }^{2}$ \\ ${ }^{1}$ Brock University, St. Catharines, Ontario, Canada, e-mail: aodesski@brocku.ca \\ ${ }^{2}$ Landau Institute for Theoretical Physics, RAS, Moscow, Russia, e-mail: sokolov@itp.ac.ru
}

\begin{abstract}
We describe the results that have so far been obtained in the classification problem for integrable $(2+1)$-dimensional systems of hydrodynamic type. The Gibbons-Tsarev (GT) systems are most fundamental here. A whole class of integrable $(2+1)$-dimensional models is related to each such system. We present the known GT systems related to algebraic curves of genus $g=0$ and $g=1$ and also a new GT system corresponding to algebraic curves of genus $g=2$. We construct a wide class of integrable models generated by the simplest GT system, which was not considered previously because it is "trivial."
\end{abstract}

Keywords: dispersionless integrable system, hydrodynamic reduction, system of Gibbons-Tsarev type 


\section{Contents}

1 Introduction $\quad 2$

2 Hydrodynamic reduction method 7

3 Gibbons-Tsarev systems

4 From GT systems to integrable models

5 Integrable weakly nonlinear (2+1)-dimensional systems $\quad \mathbf{1 9}$

5.1 The general position case ................ . . 22

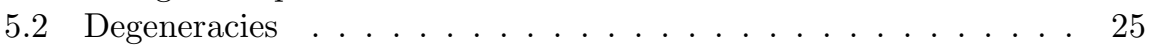

6 Discussion: Classifying integrable $(2+1)$-dimensional systems of hydrodynamic type

A Conservation laws of hydrodynamic type and integrable quasilinear second-order equations

B The GT systems and the pseudopotential representation 30

C Multidimensional integrable systems of hydrodynamic type 34

D Classifying integrable chains of hydrodynamic type 37

E List of unsolved problems

\section{Introduction}

Integrable systems play an important role in both mathematics and physics. Unfortunately, a rigorous universal definition of integrability applicable to differential equations of all types is lacking. Different views of the integrability problem were described, for example, in [1-4.

Here, we consider systems of the form

$$
\sum_{j=1}^{n} a_{i j}(\mathbf{u}) \frac{\partial u_{j}}{\partial t}+\sum_{j=1}^{n} b_{i j}(\mathbf{u}) \frac{\partial u_{j}}{\partial y}+\sum_{j=1}^{n} c_{i j}(\mathbf{u}) \frac{\partial u_{j}}{\partial x}=0, \quad \mathbf{u}=\left(u_{1}, \ldots, u_{n}\right)
$$

where $i=1, \ldots, n+k$ and $k \geq 0$, and also equations that are reducible to the above form by substitutions, for instance, quasilinear equations

$$
A_{1} Z_{t t}+A_{2} Z_{y t}+A_{3} Z_{x t}+A_{4} Z_{y y}+A_{5} Z_{x y}+A_{6} Z_{x x}=0
$$

where $A_{i}=A_{i}\left(Z_{x}, Z_{y}, Z_{t}\right)$, and equations of the form

$$
F\left(Z_{t t}, Z_{y t}, Z_{x t}, Z_{y y}, Z_{x y}, Z_{x x}\right)=0 .
$$


Here and hereafter, we use the notation $Z_{x}=\partial Z / \partial x$, etc. To include equations and systems of types (2) and (3) in our consideration, we must allow the number of equations in system (11) to exceed the number of unknowns, i.e., we must be able to consider the case $k>0$.

The existence of a dispersionless zero-curvature representation [5], 6] (also see [7] ) or (which is the same) a pseudo-potential representation is a fundamental property of integrable systems (1). This means that system (1) is equivalent to the compatibility conditions for the pair of the Hamilton-Jacobi equations of the form

$$
\Phi_{y}=A\left(\Phi_{x}, u_{1}, \ldots, u_{n}\right), \quad \Phi_{t}=B\left(\Phi_{x}, u_{1}, \ldots, u_{n}\right),
$$

where $\Phi(x, y, t)$ is a scalar function. As an example, we consider the system

$$
u_{y}=v_{x}, \quad v_{y}=u_{t}-u u_{x}
$$

We can easily verify that it admits the pseudopotential representation

$$
\Phi_{y}=\frac{\Phi_{x}^{2}}{2}+u, \quad \Phi_{t}=\frac{\Phi_{x}^{3}}{3}+u \Phi_{x}+v .
$$

Eliminating the unknown $v$, we can write system (5) as the equation

$$
u_{y y}=\left(u_{t}-u u_{x}\right)_{x}
$$

which is known as the dispersionless Kadomtsev-Petviashvili (KP) equation or as the Khokhlov-Zabolotskaya equation.

For some integrable models, the functions $A$ and $B$ in the pseudopotential representation depend on an additional spectral parameter $\lambda$. An example is the dispersionless Hirota equation

$$
a_{1} Z_{x} Z_{y t}+a_{2} Z_{y} Z_{x t}+a_{3} Z_{t} Z_{x y}=0, \quad a_{1}+a_{2}+a_{3}=0,
$$

which has the pseudopotential representation

$$
\Phi_{y}=-\frac{a_{2} \lambda}{a_{1} \lambda+a_{3}} \frac{Z_{y}}{Z_{x}} \Phi_{x}, \quad \Phi_{t}=\lambda \frac{Z_{t}}{Z_{x}} \Phi_{x}
$$

As in the case of integrable equations of the Korteveg-de Vries (KdV) equation type, the dependence on $\lambda$ can be polynomial, rational, and so on. Assuming the existence of a pseudopotential representation with a prescribed $\lambda$-dependence, we can construct examples of integrable systems (1). For small $n$, we can also classify systems admitting pseudopotential representations without the spectral parameter. But the very existence of a pseudopotential representation cannot serve as a universal integrability criterion for systems (1) because various types of pseudopotential representations exist.

The integrability of system (11) is often related to its representation as the commutation condition for a pair of vector fields [8] [11, which may or may not depend on the spectral parameter $\lambda$. If a pseudopotential representation exists, then these vector fields are Hamiltonian. Then, apparently, we cannot 
select a constructively described class of vector fields covering all the known examples and provide a rigorous universal definition of integrability based on the commutation of vector fields.

In our view, we currently have only one property of integrable systems (1D) that can be taken as a universal constructive integrability criterion, which means that we not only can verify this property for a given system but also can use it to construct new integrable models and to provide a complete classification of these systems in lower dimensions. This integrability criterion is that system (11) has a sufficient number of the so-called hydrodynamic reductions. The notion of a hydrodynamic reduction arose in 12, where the Benney chain reductions were found. It turned out that $N$-component hydrodynamic reductions can be described in terms of the compatible overdetermined Gibbons-Tsarev (GT) system

$$
\partial_{i} p_{j}=\frac{\partial_{i} u}{p_{i}-p_{j}}, \quad \partial_{i} \partial_{j} u=\frac{2 \partial_{i} u \partial_{j} u}{\left(p_{i}-p_{j}\right)^{2}}, \quad i, j=1, \ldots, N, \quad i \neq j .
$$

Here, the unknown functions $p_{1}, \ldots, p_{N}$, and $u$ depend on some variables $r^{1}, \ldots, r^{N}$ and $\partial_{i}=\partial / \partial r^{i}$. The same system (8) appears when describing hydrodynamic reductions of the Khokhlov-Zabolotskaya equation.

It was mentioned in the pioneering papers [13]-[15] that the presence of hydrodynamic reductions can be effectively used to classify integrable systems (11). Below, we briefly list the main classification results. We describe the hydrodynamic reduction method itself in Sec. 2.

The problem of classifying integrable systems of the form

$$
v_{t}+a v_{x}+p v_{y}+q w_{y}=0, \quad w_{t}+b w_{x}+r v_{y}+s w_{y}=0
$$

was considered in 14. A system of 16 nonlinear partial differential equations for the unknown coefficients $a(v, w), b(v, w), p(v, w), q(v, w), r(v, w)$, and $s(v, w)$, which was equivalent to the existence of hydrodynamic reductions, was written there. Because the obtained system for the coefficients is in involution, we can use it to study general properties of integrable systems (91). In particular, it was proved in 14 that any integrable system (9) admits a pseudopotential representation and that the space of conservation laws of hydrodynamic type is three-dimensional. The authors found many particular solutions of the system of equations for the coefficients and thus constructed new examples of integrable systems (9). But the authors did not construct the general solution explicitly.

We can easily verify that for $n=l=2$, general system (11) is reducible to canonical form (9) by a coordinate change of the form

$$
v=\psi_{1}\left(u_{1}, u_{2}\right), \quad w=\psi_{2}\left(u_{1}, u_{2}\right) .
$$

But fixing canonical form (9) destroys the group $G L(3)$ that acts on the space of integrable systems (10) by changing the independent coordinates:

$$
\bar{t}=k_{11} t+k_{12} x+k_{13} y, \bar{y}=k_{21} t+k_{22} x+k_{23} y, \bar{x}=k_{31} t+k_{32} x+k_{33} y .
$$


Reducing the action of this group to its simplest form, Odesskii [16] found another canonical form in which the system of equations for the coefficients can be solved explicitly in terms of generalized hypergeometric functions.

A special subclass of integrable equations (2) with a Lagrangian of the form $\mathcal{L}\left(Z_{t}, Z_{x}, Z_{y}\right)$ was considered in [17, [18. Necessary and sufficient conditions for the Lagrangian integrability were obtained in [17] in the form of an overdetermined system of partial differential equations for the function $\mathcal{L}$. General properties of integrable Lagrangians and new examples were also constructed. A new integrable Lagrangian $\mathcal{L}=Z_{t} Z_{x} Z_{y}$ found there is the most interesting. General integrable Lagrangians were described in $[18$ in terms of modular forms.

The general classification problem for integrable equations (2) was considered in 19, where an overdetermined system in involution for the coefficients $A_{i}\left(Z_{x}, Z_{y}, Z_{t}\right)$ (which is equivalent to the existence of hydrodynamic reductions) was written, general properties of integrable equations (2) were investigated, and new examples of these equations were found. The general solution of this overdetermined system was found in [20. It depends on five arbitrary constants and can be expressed in terms of generalized hypergeometric functions 21] of three variables after an appropriate coordinate change. We note that in addition to depending on the five essential parameters, the set of integrable equations (2) depends on 15 more parameters generated by the action of the total linear group of transformations of the parameters $Z, x, t$, and $y$, which is the invariance group for equation class (2).

Necessary and sufficient conditions for the integrability of equations of form (3) were obtained in [22]. It turned out that the function $F$ does not contain essential parameters. Moreover, interesting classes of particular solutions of the system for the function $F$ were found in [22]. The general solution was constructed in 20] in terms of the generalized hypergeometric function.

The classification problem for infinite integrable divergent chains of the form

$$
u_{1 t}=F_{1}\left(u_{1}, u_{2}\right)_{x}, u_{2 t}=F_{2}\left(u_{1}, u_{2}, u_{3}\right)_{x}, \ldots, u_{i t}=F_{i}\left(u_{1}, u_{2}, \ldots, u_{i+1}\right)_{x}, \ldots
$$

was considered in 23$]-25]$. The integrability was understood in [23] as the existence of a commuting flow of the form

$u_{1 y}=G_{1}\left(u_{1}, u_{2}, u_{3}\right)_{x}, u_{2 y}=G_{2}\left(u_{1}, u_{2}, u_{3}, u_{4}\right)_{x}, \ldots, u_{i y}=G_{i}\left(u_{1}, u_{2}, \ldots, u_{i+2}\right)_{x}, \ldots$

A necessary condition for the existence of hydrodynamic reductions (the vanishing of the Haantjes tensor) was used in 25] to produce the system of equations for the functions $F_{1}$ and $F_{2}$ that must be satisfied for the chain to be integrable.

In the above classification problems, in addition to having hydrodynamic reductions, "general position" integrable models have pseudopotential representation (4) without the spectral parameter. All the pseudopotentials arising here are related to some rational curves. Krichever constructed [26], 6] a class of pseudopotentials with arbitrarily large $n$ related to algebraic curves of an arbitrary genus $n$. It can be verified that the set of Krichever pseudopotentials 
corresponding to a rational curve does not include all the examples found when classifying system (9) for $n=2$.

The definition of the pseudopotential integrability and the complete description of integrable pseudopotentials for $n=1$ were given in terms of hydrodynamic reductions in [27. The answer was formulated in terms of hypergeometric functions.

Numerous examples related to rational curves lead to the problem of constructing a class of pseudopotentials with arbitrary $n$ that contains all these examples (possibly as reductions). Such a pseudopotential class was constructed in [16, [20. The result was expressed in terms of a special class of generalized hypergeometric functions of $n$ variables and contained $n+2$ essential parameters. When all these parameters are integers, the result can be expressed in terms of elementary functions. The problem of explicitly describing reductions of these potentials remains open. The obtained results were generalized to the case of an elliptic curve in 28 . Generalized elliptic hypergeometric functions appear in the answer in 29]; the class of elliptic hypergeometric functions there is apparently new.

Here, our presentation is based on the most fundamental notion of the hydrodynamic reduction method: the GT systems. Although such systems appeared previously [30] 34, a rigorous definition of the class of such systems, their equivalences, etc. (see Sec. 3), is apparently still lacking in the literature. We present the known GT systems related to algebraic curves of genus $g=0$ and $g=1$ and also a new GT system related to algebraic curves of genus $g=2$. A whole class of integrable (2+1)-dimensional models is related to each of the GT systems. In Sec. 4, we describe the results in [20, 28, in terms of the GT system. In Sec. 5, we construct a new broad class of integrable models generated by the simplest GT system, which was not considered previously because it is "trivial." These systems have a pseudopotential representation that depends on the spectral parameter rationally.

In Sec. 6] we share our experience acquired during several years of work with systems (11). We devote Appendices AD to conservation laws, pseudopotentials, multidimensional integrable systems (10), and integrable chains. Appendix A is important for understanding the relation between integrable systems (11) and integrable models of the type of Eqs. (2) and (3). We describe the relation between the GT systems and the pseudopotential representation without the spectral parameter in Appendix B, which is important both theoretically and technically. Examples of multidimensional integrable systems of hydrodynamic type are in Appendix [C] where we also briefly discuss how to generalize the GT-system notion to the multidimensional case. We devote Appendix D to the problem of classifying arbitrary (not necessarily divergent) hydrodynamic-type chains with the unit shift. Moreover, we present examples of integrable multidimensional chains in Appendix D. We list unsolved problems in Appendix E. 


\section{Hydrodynamic reduction method}

It is well known that integrable equations of the $\mathrm{KdV}$ equation type

$$
u_{t}=F\left(u, \frac{\partial u}{\partial x}, \ldots, \frac{\partial^{n} u}{\partial x^{n}}\right)
$$

have families of explicit solutions depending on arbitrary constants $c_{1}, \ldots, c_{N}$ for any $N$. All these finite-gap and solitonic solutions can be constructed using the so-called ODE reductions. We call a pair of compatible $N$-component systems of ODEs

$$
r_{x}^{i}=f^{i}\left(r^{1}, \ldots, r^{N}\right), \quad r_{t}^{i}=g^{i}\left(r^{1}, \ldots, r^{N}\right), \quad i=1, \ldots, N
$$

the $N$-component ODE reduction of Eq. (14) if we have a function $U\left(r^{1}, \ldots, r^{N}\right)$ such that $u=U\left(r^{1}(x, t), \ldots, r^{N}(x, t)\right)$ satisfies Eq. (14) for any solution $r^{1}(x, t), \ldots, r^{N}(x, t)$ of system (15). Clearly, a solution $u$ depends on $N$ arbitrary parameters, which are initial values for system (15) at the general position point. We can take the existence of special classes of the ODE reductions with an arbitrary $N$ as an integrability criterion for Eq. (14). For instance, we can assume that Eq. (14) admits a sequence of differential constraints of the form

$$
\frac{\partial^{m} u}{\partial x^{m}}=G_{m}\left(u, \frac{\partial u}{\partial x}, \ldots, \frac{\partial^{m-1} u}{\partial x^{m-1}}\right)
$$

with an arbitrary $m$. Clearly, we can rewrite Eq. (14) and such a differential constraint as a pair of compatible dynamical systems in the variables $x$ and $t$. Another example of an ODE reduction is the Dubrovin equations for zeros of the Baker-Akhiezer function in the $\mathrm{KdV}$ equation theory. But much more effective and constructive integrability criteria exist in the two-dimensional case. For example, we can take the existence of higher local commuting flows (higher infinitesimal symmetries) or the existence of higher conservation laws (see [35. and the references therein) as such a criterion.

Higher local symmetries and/or local conservation laws are absent if the number of independent variables is $d>2$ (see [36] for generalization of the symmetry approach to the case of nonlocal symmetries). The existence of $N$ component reductions can be considered one of the most prospective approaches for seeking new integrable models in this situation. We note that instead of the ODE reductions, we must there take some compatible systems of partial differential equations of dimension not higher than $d-1$ as reductions.

Such an approach was applied to systems of form (11) in [13, where pairs of compatible semi-Hamiltonian (see (18) below) systems of hydrodynamic type

$$
r_{t}^{i}=\lambda^{i}\left(r^{1}, \ldots, r^{N}\right) r_{x}^{i}, \quad r_{y}^{i}=\mu^{i}\left(r^{1}, \ldots, r^{N}\right) r_{x}^{i}, \quad i=1, \ldots, N,
$$

were taken as reductions. By the definition of the reduction, the corresponding solutions of system (1) are provided by some functions $u^{i}\left(r^{1}, \ldots, r^{N}\right), i=$ 
$1, \ldots, n$, that transform any solution of system (16) into solution (11). Such solutions describe the interaction of $N$ plane waves in hydrodynamics. They are sometimes called the $N$-phase solutions.

Clearly, the generalized solution of a compatible overdetermined system of form (16) contains $N$ arbitrary functions of one variable. It turns out that the functions $\lambda^{i}$ and $\mu^{i}$ in reduction (16) may contain additional functions of one variable as functional parameters; the number of these functions does not exceed $N$. The existence of hydrodynamic reductions (16) locally parameterized by $N$ functions of one variable with arbitrary $N$ was taken to be an integrability criterion for systems of form (1) in 13. The corresponding $N$-phase solutions depend on $2 N$ arbitrary functions of one variable.

The approach based on hydrodynamic reductions is universal. This means that all the currently known integrable systems (11) admit hydrodynamic reductions. All the notions in this approach can be rigorously defined (see below). It was demonstrated in 13 that the existence of hydrodynamic reductions can be verified algorithmically and can be effectively used to classify integrable cases.

We recall the definitions and basic notions of the hydrodynamic reduction method. Although they essentially coincide with those in [13, we introduce some technical improvements to make the definitions more rigorous.

The geometric theory of integrable $(1+1)$-dimensional hydrodynamic-type systems of the form

$$
r_{t}^{i}=\lambda^{i}\left(r^{1}, \ldots, r^{N}\right) r_{x}^{i}, \quad i=1, \ldots, N,
$$

was described in 37. It was shown in [38 that these systems can be integrated using the generalized hodograph method.

Definition 1 System (17) is said to be semi-Hamiltonian if we have the relations

$$
\partial_{j} \frac{\partial_{i} \lambda^{m}}{\lambda^{i}-\lambda^{m}}=\partial_{i} \frac{\partial_{j} \lambda^{m}}{\lambda^{j}-\lambda^{m}}, \quad i \neq j \neq m, \quad \partial_{i}=\frac{\partial}{\partial r^{i}} .
$$

Semi-Hamiltonian systems have an infinite number of commuting flows and conservation laws of hydrodynamic type [38], [39]. The main geometric object related to semi-Hamiltonian system (17) is the diagonal metric $g_{k k}, k=1, \ldots, N$, where

$$
\frac{1}{2} \partial_{i} \log g_{k k}=\frac{\partial_{i} \lambda^{k}}{\lambda^{i}-\lambda^{k}}, \quad i \neq k,
$$

which is called the metric associated with (17).

Definition 2 A hydrodynamic reduction of system (1) is governed by a pair of compatible semi-Hamiltonian systems of hydrodynamic type (16) and by the functions $u_{1}\left(r^{1}, \ldots, r^{N}\right), \ldots, u_{n}\left(r^{1}, \ldots, r^{N}\right)$ such that the functions

$$
u_{1}=u_{1}\left(r^{1}, \ldots, r^{N}\right), \quad \ldots, \quad u_{n}=u_{n}\left(r^{1}, \ldots, r^{N}\right)
$$

satisfy system (11) for any solution of system (16). Following [13]-[15], we say that system (11) is integrable if it admits the maximum possible number of hydrodynamic reductions. Namely, substituting (20) in (1), eliminating derivatives 
with respect to $t$ and $y$ using (16), and equating the coefficients of $r_{x}^{l}$ to zero, we obtain

$$
\begin{aligned}
& \sum_{j=1}^{n} a_{i j}(\mathbf{u}) \lambda^{l} \partial_{l} u_{j}+\sum_{j=1}^{n} b_{i j}(\mathbf{u}) \mu^{l} \partial_{l} u_{j}+\sum_{j=1}^{n} c_{i j}(\mathbf{u}) \partial_{l} u_{j}=0 \\
& i=1, \ldots, n+k, \quad l=1, \ldots, N
\end{aligned}
$$

For each $l$, we have a linear overdetermined system for $n$ unknowns $\partial_{l} u_{1}, \ldots, \partial_{l} u_{n}$ whose coefficients are independent of $l$. Because this system must have a nontrivial solution, all its $n \times n$ minors must vanish, which results in the system of algebraic equations for $\lambda^{l}$ and $\mu^{l}$, which is the same for all $l$. We assume that this system is equivalent to a single algebraic equation

$$
P\left(\lambda^{l}, \mu^{l}\right)=0
$$

(otherwise, $\lambda^{l}$ and $\mu^{l}$ are fixed, and we then have just a finite number of hydrodynamic reductions). Equation (22) determines the so-called algebraic dispersion curve. Let $p$ be a coordinate on this curve. Then Eq. (22) is equivalent to the equations

$$
\lambda^{l}=F\left(p_{l}, u_{1}, \ldots, u_{n}\right), \quad \mu^{l}=G\left(p_{l}, u_{1}, \ldots, u_{n}\right)
$$

for some functions $F$ and $G$. It was assumed in [13] that linear system (21) has a unique solution up to proportionality at a general value of $p_{l}$. Solving this system, we obtain

$$
\partial_{i} u_{m}=g_{m}\left(p_{i}, u_{1}, \ldots, u_{n}\right) \partial_{i} u_{1}, \quad m=2, \ldots, n, \quad i=1, \ldots, N,
$$

for some functions $g_{m}$. We rewrite (16) in the form

$$
r_{t}^{i}=F\left(p_{i}, u_{1}, \ldots, u_{n}\right) r_{x}^{i}, \quad r_{y}^{i}=G\left(p_{i}, u_{1}, \ldots, u_{n}\right) r_{x}^{i}, \quad i=1, \ldots, N .
$$

It is easy to see that the compatibility conditions for (24) are

$$
\frac{\partial_{i} F\left(p_{j}\right)}{F\left(p_{i}\right)-F\left(p_{j}\right)}=\frac{\partial_{i} G\left(p_{j}\right)}{G\left(p_{i}\right)-G\left(p_{j}\right)}
$$

(where we omit the arguments $u_{1}, \ldots, u_{n}$ of $F$ and $G$ ). We express $\partial_{i} p_{j}$ from (25):

$$
\partial_{i} p_{j}=f\left(p_{i}, p_{j}, u_{1}, \ldots, u_{n}\right) \partial_{i} u_{1}, \quad i \neq j, \quad i, j=1, \ldots, N .
$$

Finally, the compatibility conditions $\partial_{i} \partial_{j} u_{m}=\partial_{j} \partial_{i} u_{m}$ result in the equation

$$
\partial_{i} \partial_{j} u_{1}=h\left(p_{i}, p_{j}, u_{1}, \ldots, u_{n}\right) \partial_{i} u_{1} \partial_{j} u_{1}, \quad i \neq j, \quad i, j=1, \ldots, N .
$$

Collecting Eqs. (23), (26), and (27) together, we obtain the system of equations

$$
\begin{array}{ll}
\partial_{i} p_{j}=f\left(p_{i}, p_{j}, u_{1}, \ldots, u_{n}\right) \partial_{i} u_{1}, & i \neq j, \quad i, j=1, \ldots, N, \\
\partial_{i} u_{m}=g_{m}\left(p_{i}, u_{1}, \ldots, u_{n}\right) \partial_{i} u_{1}, & m=2, \ldots, n, \quad i=1, \ldots, N, \\
\partial_{i} \partial_{j} u_{1}=h\left(p_{i}, p_{j}, u_{1}, \ldots, u_{n}\right) \partial_{i} u_{1} \partial_{j} u_{1}, & i \neq j, \quad i, j=1, \ldots, N .
\end{array}
$$


Here, $p_{1}, \ldots, p_{N}$ and $u_{1}, \ldots, u_{n}$ are functions of $r^{1}, \ldots, r^{N}, N \geq 3$, and $\partial_{i}=$ $\partial / \partial r^{i}$. Because system (1) must have the maximum number of reductions by assumption, system (28) must be in involution (i.e., it must be completely compatible). This means that the compatibility conditions $\partial_{k} \partial_{j} p_{j}=\partial_{j} \partial_{k} p_{j}$ and $\partial_{k} \partial_{i} \partial_{j} u_{1}=\partial_{i} \partial_{k} \partial_{j} u_{1}$ are satisfied by virtue of system (28). Solutions of system (28) therefore depend on $2 N$ functions of one variable. We can take these functions to be boundary values of the Goursat problem $u_{1}\left(0, \ldots, 0, r^{i}, 0, \ldots, 0\right)$ and $p_{i}\left(0, \ldots, 0, r^{i}, 0, \ldots, 0\right)$, where $i=1, \ldots, N$.

\section{Gibbons-Tsarev systems}

In the preceding section, we showed that a system of form (28) is related to each system (1) that has the maximum set of hydrodynamic reductions.

Definition 3 A compatible system of form (28) is called an $n$-field GT system.

Definition 4 Two GT systems are said to be equivalent if they are related by a transformation of the form

$$
\begin{aligned}
& p_{i} \rightarrow \lambda\left(p_{i}, u_{1}, \ldots, u_{n}\right), \quad i=1, \ldots, N, \\
& u_{m} \rightarrow \mu_{m}\left(u_{1}, \ldots, u_{n}\right), \quad m=1, \ldots, n .
\end{aligned}
$$

Definition 5 Transformation (29), (30) is called a GT-system automorphism if it transforms this system into itself.

It is easy to see that system (28) is compatible if and only if the functions $f, g_{k}$, and $h$ satisfy a system of functional equations independent of $N$. For example, for the one-field GT system

$$
\partial_{i} p_{j}=f\left(p_{i}, p_{j}, u\right) \partial_{i} u, \quad \partial_{i} \partial_{j} u=h\left(p_{i}, p_{j}, u\right) \partial_{i} u \partial_{j} u, \quad i \neq j, \quad i, j=1, \ldots, N,
$$

these equations are

$$
\begin{aligned}
f_{u}\left(p_{2}, p_{3}, u\right)-f_{u}\left(p_{1}, p_{3}, u\right)+ & f_{p_{2}}\left(p_{2}, p_{3}, u\right) f\left(p_{1}, p_{2}, u\right)-f_{p_{1}}\left(p_{1}, p_{3}, u\right) f\left(p_{2}, p_{1}, u\right)+ \\
& +f_{p_{3}}\left(p_{2}, p_{3}, u\right) f\left(p_{1}, p_{3}, u\right)-f_{p_{3}}\left(p_{1}, p_{3}, u\right) f\left(p_{2}, p_{3}, u\right)+ \\
& +f\left(p_{2}, p_{3}, u\right) h\left(p_{1}, p_{2}, u\right)-f\left(p_{1}, p_{3}, u\right) h\left(p_{1}, p_{2}, u\right)=0 \\
h_{u}\left(p_{2}, p_{3}, u\right)-h_{u}\left(p_{1}, p_{3}, u\right)+ & h_{p_{2}}\left(p_{2}, p_{3}, u\right) f\left(p_{1}, p_{2}, u\right)-h_{p_{1}}\left(p_{1}, p_{3}, u\right) f\left(p_{2}, p_{1}, u\right)+ \\
+ & h_{p_{3}}\left(p_{2}, p_{3}, u\right) f\left(p_{1}, p_{3}, u\right)-h_{p_{3}}\left(p_{1}, p_{3}, u\right) f\left(p_{2}, p_{3}, u\right)+ \\
& +h\left(p_{2}, p_{3}, u\right) h\left(p_{1}, p_{2}, u\right)-h\left(p_{1}, p_{3}, u\right) h\left(p_{1}, p_{2}, u\right)=0
\end{aligned}
$$

\subsection{Examples of GT systems}


Example 1 Let $a_{0}, a_{1}$, and $a_{2}$ be arbitrary constants. Then the equations

$$
\begin{aligned}
\partial_{i} p_{j} & =\frac{a_{2} p_{j}^{2}+a_{1} p_{j}+a_{0}}{p_{i}-p_{j}} \partial_{i} u \\
\partial_{i} \partial_{j} u & =\frac{2 a_{2} p_{i} p_{j}+a_{1}\left(p_{i}+p_{j}\right)+2 a_{0}}{\left(p_{i}-p_{j}\right)^{2}} \partial_{i} u \partial_{j} u,
\end{aligned} \quad i, j=1, \ldots, N, \quad i \neq j,
$$

determine a one-field GT system. The original GT system (see Sec. 11) corresponds to the case where $a_{2}=a_{1}=0$ and $a_{0}=1$. In the general position case, we can use a linear transformation of the variables $p_{i}$ to reduce the system to the form

$$
\begin{aligned}
\partial_{i} p_{j} & =\frac{p_{j}\left(p_{j}-1\right)}{p_{i}-p_{j}} \partial_{i} u, \\
\partial_{i} \partial_{j} u & =\frac{2 p_{i} p_{j}-p_{i}-p_{j}}{\left(p_{i}-p_{j}\right)^{2}} \partial_{i} u \partial_{j} u,
\end{aligned} \quad i, j=1, \ldots, N, \quad i \neq j .
$$

Example 2 Let $P(x)=a_{3} x^{3}+a_{2} x^{2}+a_{1} x+a_{0}$. Then the system of equations

$$
\begin{aligned}
& \partial_{i} p_{j}=\frac{P\left(p_{j}\right)\left(p_{i}-u\right)}{P(u)\left(p_{i}-p_{j}\right)} \partial_{i} u, \\
& \partial_{i} \partial_{j} u=\frac{K_{2}\left(p_{i}, p_{j}\right) u^{2}+K_{1}\left(p_{i}, p_{j}\right) u+K_{0}\left(p_{i}, p_{j}\right)}{P(u)\left(p_{i}-p_{j}\right)^{2}} \partial_{i} u \partial_{j} u,
\end{aligned}
$$

where

$$
\begin{aligned}
& K_{2}\left(p_{i}, p_{j}\right)=2 a_{3}\left(p_{i}-p_{j}\right)^{2}, \\
& K_{1}\left(p_{i}, p_{j}\right)=-a_{3}\left(p_{i}^{2} p_{j}+p_{i} p_{j}^{2}\right)+a_{2}\left(p_{i}^{2}+p_{j}^{2}-4 p_{i} p_{j}\right)-a_{1}\left(p_{i}+p_{j}\right)-2 a_{0}, \\
& K_{0}\left(p_{i}, p_{j}\right)=2 a_{3} p_{i}^{2} p_{j}^{2}+a_{2}\left(p_{i}^{2} p_{j}+p_{i} p_{j}^{2}\right)+a_{1}\left(p_{i}^{2}+p_{j}^{2}\right)+a_{0}\left(p_{i}+p_{j}\right),
\end{aligned}
$$

is a one-field GT system. Using transformations of the form

$$
u \rightarrow \frac{a u+b}{c u+d}, \quad p_{i} \rightarrow \frac{a p_{i}+b}{c p_{i}+d},
$$

we can reduce the polynomial $P$ to one of the three canonical forms $P(x)=$ $x(x-1), P(x)=x$, or $P(x)=1$. We note that in the general position case where $P(x)=x(x-1)$, the system has an automorphism group $S_{4}$ interchanging the points $0,1, \infty$, and $u$ and generated by the elements

$$
\sigma_{1}: u \rightarrow 1-u, \quad p_{i} \rightarrow 1-p_{i}, \quad \sigma_{2}: u \rightarrow \frac{u}{u-1}, \quad p_{i} \rightarrow \frac{p_{i}}{p_{i}-1},
$$

and

$$
\sigma_{3}: u \rightarrow 1-u, \quad p_{i} \rightarrow \frac{(1-u) p_{i}}{p_{i}-u} .
$$


Example 3 We set

$$
\theta(z, \tau)=\sum_{\alpha \in \mathbb{Z}}(-1)^{\alpha} e^{2 \pi i(\alpha z+\alpha(\alpha-1) \tau / 2)}, \quad \rho(z, \tau)=\frac{\theta_{z}(z, \tau)}{\theta(z, \tau)} .
$$

Then the system

$$
\partial_{\alpha} p_{\beta}=\frac{1}{2 \pi i}\left(\rho\left(p_{\alpha}-p_{\beta}\right)-\rho\left(p_{\alpha}\right)\right) \partial_{\alpha} \tau, \quad \partial_{\alpha} \partial_{\beta} \tau=-\frac{1}{\pi i} \rho^{\prime}\left(p_{\alpha}-p_{\beta}\right) \partial_{\alpha} \tau \partial_{\beta} \tau,
$$

where $\alpha, \beta=1, \ldots, N, \alpha \neq \beta$, is a one-field GT system. The field $u$ here is the modular parameter $\tau$. We note that the same system completed with some equations with $\alpha=\beta$ appeared in [32] in relation to a very different problem.

Example 4 Let $u, v$, and $w$ be the coordinates on the moduli space of curves

$$
y^{2}=x(x-1)(x-u)(x-v)(x-w)
$$

of genus $g=2$. Then the formulas

$$
\begin{aligned}
\partial_{i} v= & \frac{v(v-1)\left(p_{i}-u\right)}{u(u-1)\left(p_{i}-v\right)} \partial_{i} u \\
\partial_{i} w= & \frac{w(w-1)\left(p_{i}-u\right)}{u(u-1)\left(p_{i}-w\right)} \partial_{i} u \\
\partial_{i} p_{j}= & \frac{p_{j}\left(p_{j}-1\right)\left(p_{i}-u\right)\left(p_{i}-v\right)\left(p_{i}-w\right)+y\left(p_{i}\right) y\left(p_{j}\right)}{u(u-1)\left(p_{i}-v\right)\left(p_{i}-w\right)\left(p_{i}-p_{j}\right)} \partial_{i} u \\
\partial_{i} \partial_{j} u= & \left(\frac{u\left(p_{i}+p_{j}\right)+(u-1)\left(p_{i}^{2}+p_{j}^{2}\right)+\left(p_{i}+p_{j}-4 u\right) p_{i} p_{j}}{u(u-1)\left(p_{i}-p_{j}\right)^{2}}+\right. \\
& \left.\quad+\frac{\left(2 v w-\left(p_{i}+p_{j}\right)(v+w)+2 p_{i} p_{j}\right) y\left(p_{i}\right) y\left(p_{j}\right)}{u(u-1)\left(p_{i}-v\right)\left(p_{i}-w\right)\left(p_{j}-v\right)\left(p_{j}-w\right)\left(p_{i}-p_{j}\right)^{2}}\right) \partial_{i} u \partial_{j} u
\end{aligned}
$$

determine a three-field GT system.

The systems in Examples 3 and 4 are related to the moduli spaces of curves of the respective genera $g=1$ and $g=2$. If we parameterize elliptic curves using the formula $y^{2}=x(x-1)(x-u)$ instead of the modular parameter, then we can rewrite the system in Example 3 in a form similar to the system in Example 4

The functions $f$ and $h$ have poles at $p_{i}=p_{j}$ in Examples 14. But there are GT systems holomorphic at $p_{i}=p_{j}$.

Example 5 The formulas

$$
\partial_{i} p_{j}=0, \quad \partial_{i} u_{m}=g_{m}\left(p_{i}\right) \partial_{i} u_{1}, \quad \partial_{i} \partial_{j} u_{1}=0
$$

define an $n$-field $G T$ system for any $n$ and for arbitrary functions $g_{m}(x)$. 


\subsection{GT-system extensions}

Definition 6 An extension of GT system (28) using the field functions $u_{n+1}, \ldots, u_{n+m}$ is an auxiliary system of the form

$$
\partial_{i} u_{k}=g_{k}\left(p_{i}, u_{1}, \ldots, u_{n+m}\right) \partial_{i} u_{1}, \quad k=n+1, \ldots, n+m, \quad i=1, \ldots, N,
$$

such that Eqs. (28) and (38) are compatible.

Example 1a (continuation of Example11). System (33) admits the one-field extensions

$$
\begin{aligned}
& \partial_{i} v=\frac{v(v-1)}{p_{i}-v} \partial_{i} u, \\
& \partial_{i} v=\left(\frac{1}{p_{i}^{2}}+\frac{v}{p_{i}}\right) \partial_{i} u, \\
& \partial_{i} v=\left(\frac{1}{\left(p_{i}-1\right)^{2}}-\frac{v}{p_{i}-1}\right) \partial_{i} u, \\
& \partial_{i} v=\left(\frac{c_{1}}{p_{i}-1}+\frac{c_{2}}{p_{i}}+\left(c_{3} e^{u}-c_{4} e^{2 u}\right) p_{i}+c_{4} e^{2 u} p_{i}^{2}\right) \partial_{i} u,
\end{aligned}
$$

where $c_{i}$ are arbitrary constants.

Describing all extensions for a given GT system is a complicated problem. But extensions of type (39) always exist and can be constructed in a uniform way. Namely, it turns out that if we complete system (28) with the equation

$$
\partial_{i} u_{n+1}=f\left(p_{i}, u_{n+1}, u_{1}, \ldots, u_{n}\right) \partial_{i} u_{1}
$$

(the function $f$ is the same as in (28)), then the obtained $(n+1)$-field system remains compatible. We call this procedure the regular extension. For example, the regular extension in the case of Example1 1 results in the additional equation

$$
\partial_{i} v=\frac{a_{2} v^{2}+a_{1} v+a_{0}}{p_{i}-v} \partial_{i} u .
$$

We note that if we take $v$ as a new field variable, then the first formula in Example 1 becomes

$$
\partial_{i} p_{j}=\frac{\left(a_{2} p_{j}^{2}+a_{1} p_{j}+a_{0}\right)\left(p_{i}-v\right)}{\left(a_{2} v^{2}+a_{1} v+a_{0}\right)\left(p_{i}-p_{j}\right)} \partial_{i} v, \quad i, j=1, \ldots, N, \quad i \neq j .
$$

We see that we can use this trick to obtain the canonical forms of the GT system in Example 2 from the corresponding GT systems in Example 1.

Clearly, we can apply the regular extension procedure repeatedly. For example, the $n$-fold regular extension of GT system (8) is

$\partial_{i} p_{j}=\frac{1}{p_{i}-p_{j}} \partial_{i} w, \quad \partial_{i} \partial_{j} w=\frac{2}{\left(p_{i}-p_{j}\right)^{2}} \partial_{i} w \partial_{j} w, \quad \partial_{i} u_{k}=\frac{\partial_{i} w}{p_{i}-u_{k}}, \quad k=1, \ldots, n$. 
For this system, we can construct degenerations corresponding to merging some fields. In particular, merging all the fields $u_{i} \rightarrow w, i=1, \ldots, n$, results in the GT system

$$
\begin{aligned}
\partial_{i} p_{j} & =\frac{1}{p_{i}-p_{j}} \partial_{i} w_{1}, \quad \partial_{i} \partial_{j} w_{1}=\frac{2}{\left(p_{i}-p_{j}\right)^{2}} \partial_{i} w_{1} \partial_{j} w_{1}, \\
\partial_{i} w_{j} & =\left(p_{i}^{j-1}-\sum_{k=1}^{j-2} k u_{k} p_{i}^{j-k-2}\right) \partial_{i} w_{1} .
\end{aligned}
$$

This extension at $n=1$ exactly coincides with the GT system for dispersionless KP equation (5) (see Example 6] below).

We note that we cannot obtain the GT system in Example 4 from a system with fewer fields using a regular extension procedure.

\section{From GT systems to integrable models}

Constructing compatible families of systems of form (24) is our next step on the path from GT systems to integrable models (1).

4.1. Families of $(1+1)$-dimensional hydrodynamic-type systems associated with a GT system

Definition 7 A family of semi-Hamiltonian systems of hydrodynamic type

$$
r_{t}^{i}=F\left(p_{i}, u_{1}, \ldots, u_{n}\right) r_{x}^{i}
$$

parameterized by solutions $p_{i}\left(r^{1}, \ldots, r^{N}\right), i=1, \ldots, N, u_{j}\left(r^{1}, \ldots, r^{N}\right), j=$ $1, \ldots, n$, of $G T$ system (28) is called a family of $(1+1)$-dimensional systems associated with system (28).

Two (1+1)-dimensional systems (24) are compatible if and only if the functions $F$ and $G$ satisfy functional equation (25). This equation has trivial solutions $F$ and $G=c_{1} F+c_{2}$, where $F$ is an arbitrary function and $c_{i}$ are arbitrary constants.

Not every GT system admits nontrivial solutions $F$ and $G$ of Eq. (25) depending essentially on the field functions $u_{i}$. For example, such solutions exist only under a special choice of the functions $g_{m}$ in Example 5

We consider one-field GT systems (31). In accordance with (25), we must then have functions of two variables $F(x, u)$ and $G(x, u)$ such that

$$
\frac{f(x, y, u) \frac{\partial F}{\partial y}(y, u)+\frac{\partial F}{\partial u}(y, u)}{F(x, u)-F(y, u)}=\frac{f(x, y, u) \frac{\partial G}{\partial y}(y, u)+\frac{\partial G}{\partial u}(y, u)}{G(x, u)-G(y, u)} .
$$

Proposition 1 Every one-field GT system admitting a nontrivial solution $F(x, u)$, $G(x, u)$ of Eq. (44) is equivalent to a system in either Example 1 or Example 2 
The following results on constructing solutions of functional equation (44) for $n$-field systems with arbitrary $n$ were obtained in [20], 28].

Example 1b (continuation of Example 1). An $n$-fold application of the regular extension procedure to GT system (33) with a quadratic polynomial of general position results in the GT system

$$
\begin{aligned}
& \partial_{i} p_{j}=\frac{p_{j}\left(p_{j}-1\right)}{p_{i}-p_{j}} \partial_{i} w, \quad \partial_{i} \partial_{j} w=\frac{2 p_{i} p_{j}-p_{i}-p_{j}}{\left(p_{i}-p_{j}\right)^{2}} \partial_{i} w \partial_{j} w, \\
& i, j=1, \ldots, N, \quad i \neq j, \\
& \partial_{i} u_{j}=\frac{u_{j}\left(u_{j}-1\right) \partial_{i} w}{p_{i}-u_{j}}, \quad i=1, \ldots, N, \quad j=1, \ldots, n .
\end{aligned}
$$

We consider the compatible overdetermined system of partial differential equations

$$
\begin{aligned}
\frac{\partial^{2} h}{\partial u_{j} \partial u_{k}}= & \frac{s_{j}}{u_{j}-u_{k}} \frac{\partial h}{\partial u_{k}}+\frac{s_{k}}{u_{k}-u_{j}} \frac{\partial h}{\partial u_{j}}, \quad i, j=1, \ldots, n, \quad j \neq k \\
\frac{\partial^{2} h}{\partial u_{j} \partial u_{j}}= & -\left(1+\sum_{k=1}^{n+2} s_{k}\right) \frac{s_{j}}{u_{j}\left(u_{j}-1\right)} h+\frac{s_{j}}{u_{j}\left(u_{j}-1\right)} \sum_{\substack{k=1, k \neq j}}^{n} \frac{u_{k}\left(u_{k}-1\right)}{u_{k}-u_{j}} \frac{\partial h}{\partial u_{k}}+ \\
& +\left(\sum_{\substack{k=1 \\
k \neq j}}^{n} \frac{s_{k}}{u_{j}-u_{k}}+\frac{s_{j}+s_{n+1}}{u_{j}}+\frac{s_{j}+s_{n+2}}{u_{j}-1}\right) \frac{\partial h}{\partial u_{j}},
\end{aligned}
$$

where $s_{1}, \ldots, s_{n+2} \in \mathbb{C}$ are arbitrary parameters. Solutions of this system belong to the class of generalized hypergeometric functions [21]. We can easily see that the vector space $\mathcal{H}$ of all the solutions of this system has the dimension $n+1$. For any $h \in \mathcal{H}$, we set

$S(h, p)=\sum_{i=1}^{n} u_{i}\left(u_{i}-1\right)\left(p-u_{1}\right) \cdots \hat{i} \cdots\left(p-u_{n}\right) h_{u_{i}}+\left(1+\sum_{i=1}^{n+2} s_{i}\right)\left(p-u_{1}\right) \cdots\left(p-u_{n}\right) h$,

where $\hat{i}$ means that we omit the corresponding multiplier in the product. The function $S$ is obviously a polynomial of degree $n$ in $p$.

Let $h_{1}, h_{2}$, and $h_{3}$ be linearly independent functions in $\mathcal{H}$. Then the functions

$$
F=\frac{S\left(h_{1}, p\right)}{S\left(h_{3}, p\right)}, \quad G=\frac{S\left(h_{2}, p\right)}{S\left(h_{3}, p\right)}
$$

satisfy functional equation (25).

Moreover, we have solutions of Eq. (25) of form (50) for which $S$ has a degree less than $n$. In this case, the polynomial $S$ is to be defined in a more complicate 
way. Namely, we fix $k$ linearly independent functions $h_{1}, \ldots, h_{k} \in \mathcal{H}$. Then for each $h \in \mathcal{H}$, the polynomial $S(h, p)$ is given by the formula

$$
S(h, p)=\frac{1}{\Delta} \sum_{i=1}^{n-k+1} u_{i}\left(u_{i}-1\right)\left(p-u_{1}\right) \cdots \hat{i} \cdots\left(p-u_{n-k+1}\right) \Delta_{i}(h),
$$

where $h \in \mathcal{H}$ and

$$
\begin{aligned}
& \Delta=\operatorname{det}\left(\begin{array}{cccc}
h_{1} & h_{2} & \cdots & h_{k} \\
h_{1, u_{n-k+2}} & h_{2, u_{n-k+2}} & \cdots & h_{k, u_{n-k+2}} \\
\vdots & \vdots & \ddots & \vdots \\
h_{1, u_{n}} & h_{2, u_{n}} & \cdots & h_{k, u_{n}}
\end{array}\right) \text {, } \\
& \Delta_{i}(h)=\operatorname{det}\left(\begin{array}{ccccc}
h & h_{1} & h_{2} & \cdots & h_{k} \\
h_{u_{i}} & h_{1, u_{i}} & h_{2, u_{i}} & \cdots & h_{k, u_{i}} \\
h_{u_{n-k+2}} & h_{1, u_{n-k+2}} & h_{2, u_{n-k+2}} & \cdots & h_{k, u_{n-k+2}} \\
\vdots & \vdots & \vdots & \ddots & \vdots \\
h_{u_{n}} & h_{1, u_{n}} & h_{2, u_{n}} & \cdots & h_{k, u_{n}}
\end{array}\right) .
\end{aligned}
$$

Clearly, $S(h, p)$ is a polynomial of degree $n-k$ in $p$. We note that linear transformations $h_{i} \rightarrow c_{i 1} h_{1}+\cdots+c_{i k} h_{k}$ and $h \rightarrow h+d_{1} h_{1}+\cdots+d_{k} h_{k}$ with constant coefficients $c_{i j}$ and $d_{i}$ do not change $S(h, p)$.

There are solutions of functional equation (25) for GT system (45), (46) different from those constructed above. For example, for $n=2$, we have the solution

$$
F=\frac{\left(p-u_{1}\right)\left(p-u_{2}\right)}{p\left(u_{1}-u_{2}\right)}, \quad G=\frac{\left(p-u_{1}\right)\left(p-u_{2}\right)}{(p-1)\left(u_{1}-u_{2}\right)} .
$$

Apparently, these solutions are related to singular rational algebraic curves.

Example 3a (continuation of Example 3). A regular $n$-field extension of GT system (35) is given by the formulas

$$
\partial_{\alpha} u_{\beta}=\frac{1}{2 \pi i}\left(\rho\left(p_{\alpha}-u_{\beta}\right)-\rho\left(p_{\alpha}\right)\right) \partial_{\alpha} \tau, \quad \beta=1, \ldots, n .
$$

A nontrivial solution of functional equation (25) is given by formulas (50) with

$$
S(h, p)=\sum_{\alpha=1}^{n} \frac{\theta\left(u_{\alpha}\right) \theta\left(p-u_{\alpha}-\eta\right)}{\theta\left(u_{\alpha}+\eta\right) \theta\left(p-u_{\alpha}\right)} h_{u_{\alpha}}-\left(s_{1}+\cdots+s_{n}\right) \frac{\theta^{\prime}(0) \theta(p-\eta)}{\theta(\eta) \theta(p)} h .
$$


Here, $\eta=s_{1} u_{1}+\cdots+s_{n} u_{n}+r \tau+\eta_{0}$, where $s_{1}, \ldots, s_{n}, r, \eta_{0}$ are arbitrary constants and $h\left(u_{1}, \ldots, u_{n}, \tau\right)$ is a solution of the elliptic hypergeometric system

$$
\begin{aligned}
& h_{u_{\alpha} u_{\beta}}= s_{\beta}\left(\rho\left(u_{\beta}-u_{\alpha}\right)+\rho\left(u_{\alpha}+\eta\right)-\rho\left(u_{\beta}\right)-\rho(\eta)\right) h_{u_{\alpha}}+ \\
&+s_{\alpha}\left(\rho\left(u_{\alpha}-u_{\beta}\right)+\rho\left(u_{\beta}+\eta\right)-\rho\left(u_{\alpha}\right)-\rho(\eta)\right) h_{u_{\beta}}, \\
& h_{u_{\alpha} u_{\alpha}}= s_{\alpha} \sum_{\beta \neq \alpha}\left(\rho\left(u_{\alpha}\right)+\rho(\eta)-\rho\left(u_{\alpha}-u_{\beta}\right)-\rho\left(u_{\beta}+\eta\right)\right) h_{u_{\beta}}+ \\
&+\left(\sum_{\beta \neq \alpha} s_{\beta} \rho\left(u_{\alpha}-u_{\beta}\right)+\left(s_{\alpha}+1\right) \rho\left(u_{\alpha}+\eta\right)+s_{\alpha} \rho(-\eta)+\right. \\
&\left.+\left(s_{0}-s_{\alpha}-1\right) \rho\left(u_{\alpha}\right)+2 \pi i r\right) h_{u_{\alpha}}-s_{0} s_{\alpha}\left(\rho^{\prime}\left(u_{\alpha}\right)-\rho^{\prime}(\eta)\right) h, \\
& h_{\tau}=\frac{1}{2 \pi i} \sum_{\beta}\left(\rho\left(u_{\beta}+\eta\right)-\rho(\eta)\right) h_{u_{\beta}}-\frac{s_{0}}{2 \pi i} \rho^{\prime}(\eta) h .
\end{aligned}
$$

We can show that the solution space of this system has the dimension $n+1$.

We construct elliptic analogues of solutions (50), (51) as follows. We fix $k$ linearly independent solutions $h_{1}, \ldots, h_{k}$ of the elliptic hypergeometric system. For any solution $h$, we then have

$S(h, p)=\frac{1}{\Delta}\left(\sum_{\alpha=1}^{n-k} \frac{\theta\left(u_{\alpha}\right) \theta\left(p-u_{\alpha}-\eta\right)}{\theta\left(u_{\alpha}+\eta\right) \theta\left(p-u_{\alpha}\right)} \Delta_{\alpha}(h)-\left(s_{1}+\cdots+s_{n}\right) \frac{\theta^{\prime}(0) \theta(p-\eta)}{\theta(\eta) \theta(p)} \Delta_{0}(h)\right)$,

where

$$
\begin{gathered}
\Delta=\operatorname{det}\left(\begin{array}{ccc}
h_{1, u_{n-k+1}} & \cdots & h_{k, u_{n-k+1}} \\
\vdots & \ddots & \vdots \\
h_{1, u_{n}} & \cdots & h_{k, u_{n}}
\end{array}\right), \\
\Delta_{\alpha}(h)=\operatorname{det}\left(\begin{array}{cccc}
h_{u_{\alpha}} & h_{1, u_{\alpha}} & \cdots & h_{k, u_{\alpha}} \\
h_{u_{n-k+1}} & h_{1, u_{n-k+1}} & \cdots & h_{k, u_{n-k+1}} \\
\vdots & \vdots & \ddots & \vdots \\
h_{u_{n}} & h_{1, u_{n}} & \cdots & h_{k, u_{n}}
\end{array}\right), \\
\Delta_{0}(h)=\operatorname{det}\left(\begin{array}{cccc}
h & h_{1} & \cdots & h_{k} \\
h_{u_{n-k+1}} & h_{1, u_{n-k+1}} & \cdots & h_{k, u_{n-k+1}} \\
\vdots & \vdots & \ddots & \vdots \\
h_{u_{n}} & h_{1, u_{n}} & \cdots & h_{k, u_{n}}
\end{array}\right) .
\end{gathered}
$$

4.2. Integrable three-dimensional systems generated by a pair of compatible families. Given GT system (28) and a pair of compatible fami- 
lies (24) (i.e., a solution $F, G$ of functional equation (25)), we can construct the corresponding system (1) by a simple calculation.

Lemma We consider the linear space $V$ of functions of the variable p generated by $3 n$ functions

$\left\{F\left(p, u_{1}, \ldots, u_{n}\right) g_{j}\left(p, u_{1}, \ldots, u_{n}\right), G\left(p, u_{1}, \ldots, u_{n}\right) g_{j}\left(p, u_{1}, \ldots, u_{n}\right), g_{j}\left(p, u_{1}, \ldots, u_{n}\right) ; j=1, \ldots, n\right\}$.

Here, $g_{1}=1$ by definition. The system of form (11) with hydrodynamic reductions (24) then consists of $n+k$ equations if and only if the dimension of $V$ is $2 n-k$. The coefficients of system (1) are determined by the relations

$$
\sum_{j=1}^{n}\left(a_{i j}(\mathbf{u}) F\left(p, u_{1}, \ldots, u_{n}\right)+b_{i j}(\mathbf{u}) G\left(p, u_{1}, \ldots, u_{n}\right)+c_{i j}(\mathbf{u})\right) g_{j}\left(p, u_{1}, \ldots, u_{n}\right)=0
$$

$i=1, \ldots, n+k$.

Corollary If the functions $F$ and $G$ are determined by formulas of type (50), where the expression $S(h, p)$ is linear in $h$ and $h_{1}, h_{2}$, and $h_{3}$ are arbitrary elements of a vector space, then the linear transformation $\bar{h}_{i}=\sum_{j=1}^{3} k_{i j} h_{j}$ corresponds to transformation (11) in the corresponding system (11).

Example 6 We consider the extension $\partial_{i} v=p_{i} \partial_{i} u$ of GT system (8). The corresponding functional equation (44) has the solution $F=p^{2}+u, G=p$. Moreover, we have $g_{1}=1$ and $g_{2}=p$. The space generated by the set of functions $\left\{p^{2}+u, p^{3}+u p, p, p^{2}, 1, p\right\}$ has the dimension $\alpha=4$. The matrices $A, B$, and $C$ composed of the unknown coefficients of system (11) are determined up to simultaneous multiplication by an arbitrary matrix from the left. We can easily verify that using such a transformation, we can reduce any solution of the system of eight equations, which is equivalent to relations (54), to $a_{11}=1$, $a_{12}=a_{21}=a_{22}=0, b_{11}=b_{22}=0, b_{12}=-1, b_{21}=1, c_{11}=-u, c_{12}=c_{21}=0$, and $c_{22}=-1$. This solution corresponds to system (5). 
An analogous calculation for (45), (46), (49), and (50) results in the system for $u_{1}, \ldots, u_{n}$ :

$$
\begin{gathered}
\sum_{i \neq j}\left(h_{q, u_{j}} h_{r, u_{i}}-h_{r, u_{j}} h_{q, u_{i}}\right) \frac{u_{j}\left(u_{j}-1\right) u_{i, t_{s}}-u_{i}\left(u_{i}-1\right) u_{j, t_{s}}}{u_{j}-u_{i}}+ \\
+\sigma\left(h_{q} h_{r, u_{j}}-h_{r} h_{q, u_{j}}\right) u_{j, t_{s}}+ \\
+\sum_{i \neq j}\left(h_{r, u_{j}} h_{s, u_{i}}-h_{s, u_{j}} h_{r, u_{i}}\right) \frac{u_{j}\left(u_{j}-1\right) u_{i, t_{q}}-u_{i}\left(u_{i}-1\right) u_{j, t_{q}}}{u_{j}-u_{i}}+ \\
+\sigma\left(h_{r} h_{s, u_{j}}-h_{s} h_{r, u_{j}}\right) u_{j, t_{q}}+ \\
+\sum_{i \neq j}\left(h_{s, u_{j}} h_{q, u_{i}}-h_{q, u_{j}} h_{s, u_{i}}\right) \frac{u_{j}\left(u_{j}-1\right) u_{i, t_{r}}-u_{i}\left(u_{i}-1\right) u_{j, t_{r}}}{u_{j}-u_{i}}+ \\
+\sigma\left(h_{s} h_{q, u_{j}}-h_{q} h_{s, u_{j}}\right) u_{j, t_{r}}=0,
\end{gathered}
$$

where $j=1, \ldots, n$ and $\sigma=1+s_{1}+\cdots+s_{n+2}$. Here, the subscript after the comma indicates the partial derivative with respect to the corresponding argument, $q=0, r=1, s=2$ and $t_{0}=x, t_{1}=t, t_{2}=y$ in expressions like $h_{q, u_{j}}$ and $u_{i, t_{q}}$.

Remark Let $h_{0}, h_{1}, h_{2}, \ldots, h_{n}$ be a basis in the space of solutions of hypergeometric system (47), (48). We associate the proper time $t_{i}$ with each $h_{i}$. For each triple of pairwise distinct $0 \leq q, r, s \leq n$, formula (55) defines the corresponding three-dimensional system with the times $t_{q}, t_{r}$, and $t_{s}$. According to [20], all these systems are mutually compatible.

The explicit form of systems related to the case $k>0$ can be found in [20]. Systems generated by the elliptic GT system in Example 3 were presented in [28].

\section{Integrable weakly nonlinear $(2+1)$-dimensional systems}

The functions $f$ and $h$ have a pole on the diagonal $p_{i}=p_{j}$ in all GT-system examples (28) above. But we also have GT systems holomorphic at $p_{i}=p_{j}$. In particular, such are the systems in Example 5.

Definition 8 Integrable system (11) is said to be weakly nonlinear if the corresponding GT system is holomorphic on the diagonal $p_{i}=p_{j}$.

The GT-system holomorphicity conditions are expressed as follows in terms of the solution $F, G$ of functional equation (25). Writing (25) in the form

$$
\left(\partial_{i} p_{j} F^{\prime}\left(p_{j}\right)+\sum_{\alpha} F\left(p_{j}\right)_{u_{\alpha}} \partial_{i} u_{\alpha}\right)\left(G\left(p_{i}\right)-G\left(p_{j}\right)\right)=\left(\partial_{i} p_{j} G^{\prime}\left(p_{j}\right)+\sum_{\alpha} G\left(p_{j}\right)_{u_{\alpha}} \partial_{i} u_{\alpha}\right)\left(F\left(p_{i}\right)-F\left(p_{j}\right)\right),
$$


where the prime denotes the derivative with respect to $p_{j}$, and expressing $\partial_{i} p_{j}$ from it, we find that this expression is holomorphic on the diagonal if and only if

$$
\sum_{\alpha}\left(F\left(p_{i}\right)_{u_{\alpha}} G^{\prime}\left(p_{i}\right)-G\left(p_{i}\right)_{u_{\alpha}} F^{\prime}\left(p_{i}\right)\right) \partial_{i} u_{\alpha}=0 .
$$

Proposition 2 Let system (11) be weakly nonlinear, and let $k=0$ (i.e., the number of unknowns is equal to the number of equations in the system). Then the matrix

$$
\tilde{A}=\left(a_{11} A+a_{12} B+a_{13} C\right)^{-1}\left(a_{21} A+a_{22} B+a_{23} C\right)
$$

is linearly degenerate for all constant $a_{i j}$.

We recall that a matrix $P\left(u_{1}, \ldots, u_{n}\right)$ is said to be linearly degenerate if the derivative of any eigenvalue along the corresponding eigenvector vanishes.

Proof Using substitution (29), we reduce $G(p)$ to the form $G=p$. Then (56) becomes

$$
\sum_{\alpha} F\left(p_{i}\right)_{u_{\alpha}} \partial_{i} u_{\alpha}=0
$$

An arbitrary change of variables (11) brings system (1) to the form $\mathbf{u}_{t}=\tilde{A} \mathbf{u}_{x}+$ $\widetilde{B} \mathbf{u}_{y}$, where

$$
\begin{aligned}
& \tilde{A}=\left(a_{11} A+a_{12} B+a_{13} C\right)^{-1}\left(a_{21} A+a_{22} B+a_{23} C\right), \\
& \widetilde{B}=\left(a_{11} A+a_{12} B+a_{13} C\right)^{-1}\left(a_{31} A+a_{32} B+a_{33} C\right) .
\end{aligned}
$$

For $\mu^{l}=p_{l}$ and $\lambda^{l}=F\left(p_{l}\right)$, formula (21) for this system yields

$$
\left(\tilde{A}+p_{i} \widetilde{B}-F\left(p_{i}\right) \operatorname{Id}\right)\left(\partial_{i} u_{1}, \ldots, \partial_{i} u_{n}\right)^{\mathrm{T}}=0 .
$$

For this system, formula (57) implies that the derivative of the eigenvalue $F\left(p_{i}\right)$ along the eigenvector $\left(\partial_{i} u_{1}, \ldots, \partial_{i} u_{n}\right)$ vanishes. The matrix $\tilde{A}+p_{i} \widetilde{B}$ is therefore linearly degenerate at any value of the parameter $p_{i}$.

The obtained result states that if the number of equations in system (11) is equal to the number of unknown functions, then every two-dimensional system describing solutions $\mathbf{u}=\mathbf{u}\left(c_{1} x+c_{2} y+c_{3} t, c_{4} x+c_{5} y+c_{6} t\right)$ of the traveling-wave type for weakly nonlinear system (11) is weakly nonlinear in the sense in [40].

Example 7 We consider the two-component system (see [13])

$$
\left(\begin{array}{c}
v_{t} \\
w_{t}
\end{array}\right)+\left(\begin{array}{ll}
a & 0 \\
0 & b
\end{array}\right)\left(\begin{array}{c}
v_{x} \\
w_{x}
\end{array}\right)+\left(\begin{array}{ll}
p & q \\
r & s
\end{array}\right)\left(\begin{array}{c}
v_{y} \\
w_{y}
\end{array}\right)=0,
$$


where

$$
\begin{aligned}
& a=w, \quad b=v, \quad r=\frac{P(w)}{w-v}, \quad q=\frac{P(v)}{v-w}, \\
& s=\frac{P(v)}{w-v}+\frac{1}{3} P^{\prime}(v), \quad p=\frac{P(w)}{v-w}+\frac{1}{3} P^{\prime}(w) .
\end{aligned}
$$

Here, $P$ is an arbitrary third-degree polynomial. The corresponding GT system

$$
\begin{gathered}
\partial_{i} p_{j}=\frac{P(w)}{(w-v) P(v)} p_{j}^{2} p_{i}+\left(\frac{1}{w-v}+\frac{P^{\prime}(v)}{P(v)}\right) p_{j} p_{i}- \\
-\left(\frac{1}{v-w}+\frac{P^{\prime}(w)}{P(w)}\right) p_{j}-\frac{P(v)}{(v-w) P(w)}, \\
\partial_{i} v=p_{i} \partial_{i} w \\
\partial_{i} \partial_{j} w=\left(\frac{P(w)}{(v-w) P(v)} p_{i} p_{j}+\frac{1}{v-w}+\frac{P^{\prime}(w)}{P(w)}\right) \partial_{i} w \partial_{j} w
\end{gathered}
$$

is polynomial in $p_{i}$ and $p_{j}$, and the initial $(2+1)$-dimensional system is therefore weakly nonlinear. We can verify that this GT system is equivalent to the GT system

$$
\partial_{i} p_{j}=0, \quad \partial_{i} u_{2}=p_{i} \partial_{i} u_{1}, \quad \partial_{i} \partial_{j} u_{1}=0,
$$

which belongs to the class in Example 5 .

As shown in [13], system (9) has a linear pseudopotential representation with the spectral parameter. This turns out to be a general property of systems (37) in Example 5.

Proposition 3 Let $F\left(p, u_{1}, \ldots, u_{n}\right)$ and $G\left(p, u_{1}, \ldots, u_{n}\right)$ be a solution of functional equation (25) for a GT system of form (37). Then the corresponding $(2+1)$-dimensional system admits the pseudopotential representation

$$
\psi_{t}=F\left(\lambda, u_{1}, \ldots, u_{n}\right) \psi_{x}, \quad \psi_{y}=G\left(\lambda, u_{1}, \ldots, u_{n}\right) \psi_{x},
$$

where $\lambda$ is the spectral parameter.

Proof From (25) with the vanishing of $\partial_{i} p_{j}$ taken into account, we obtain

$$
\frac{\sum_{\alpha} F\left(p_{j}\right)_{u_{\alpha}} \partial_{i} u_{\alpha}}{F\left(p_{i}\right)-F\left(p_{j}\right)}=\frac{\sum_{\alpha} G\left(p_{j}\right)_{u_{\alpha}} \partial_{i} u_{\alpha}}{G\left(p_{i}\right)-G\left(p_{j}\right)} .
$$

On the other hand, the compatibility condition for system (59) is $F_{y}+F G_{x}=$ $G_{t}+G F_{x}$, whence we obtain

$$
\sum_{\alpha}\left(F(\lambda)_{u_{\alpha}} u_{\alpha y}+F(\lambda) G(\lambda)_{u_{\alpha}} u_{\alpha x}\right)=\sum_{\alpha}\left(G(\lambda)_{u_{\alpha}} u_{\alpha t}+G(\lambda) F(\lambda)_{u_{\alpha}} u_{\alpha x}\right) .
$$


Substituting the expressions

$$
u_{\alpha y}=\sum_{i} \partial_{i} u_{\alpha} r_{y}^{i}=\sum_{i} \partial_{i} u_{\alpha} G\left(p_{i}\right) r_{x}^{i}, \quad u_{\alpha t}=\sum_{i} \partial_{i} u_{\alpha} F\left(p_{i}\right) r_{x}^{i}
$$

in this equality and replacing $\lambda$ with $p_{j}$, we obtain (60).

\subsection{The general position case}

Using our observation on the equivalence between GT system (58) and a system of form (37) with rational functions $g_{m}$, we now generalize Example 7 to the case of arbitrary $n$ and $k$.

We consider an $(n+1)$-field GT system

$$
\partial_{i} p_{j}=0, \quad \partial_{i} u_{m}=\frac{\lambda_{m}-\lambda_{0}}{p_{i}-\lambda_{m}} \partial_{i} w, \quad \partial_{i} \partial_{j} w=0
$$

where $u_{1}, \ldots, u_{n}$ and $w$ are the field functions and $\lambda_{0}, \lambda_{1}, \ldots, \lambda_{n}$ are the parameters. We let $\mathcal{H}_{n}$ denote the linear space of functions in the variables $u_{1}, \ldots, u_{n}$ generated by the elements $1, e^{u_{1}}, \ldots, e^{u_{n}}$. For any function $g=$ $a_{0}+a_{1} e^{u_{1}}+\cdots+a_{n} e^{u_{n}} \in \mathcal{H}_{n}$, we set

$$
S_{n}(g, p)=\frac{a_{0}}{p-\lambda_{0}}+\sum_{i=1}^{n} \frac{a_{i} e^{u_{i}}}{p-\lambda_{i}} .
$$

For any $k \in \mathbb{N}$ such that $0<k<n-1$, we fix the functions $h_{1}, \ldots, h_{k} \in \mathcal{H}_{n}$, where $h_{i}=b_{i, 0}+b_{i, 1} e^{u_{1}}+\cdots+{ }_{i, n} e^{u_{n}}$, and set

$$
S_{n, k}(g, p)=\operatorname{det}\left(\begin{array}{ccccc}
S_{n}(g, p) & S_{n}\left(h_{1}, p\right) & S_{n}\left(h_{2}, p\right) & \cdots & S_{n}\left(h_{k}, p\right) \\
g & h_{1} & h_{2} & \cdots & h_{k} \\
a_{n-k+2} & b_{1, n-k+2} & b_{2, n-k+2} & \cdots & b_{k, n-k+2} \\
\vdots & \vdots & \vdots & \ddots & \vdots \\
a_{n} & b_{1, n} & b_{2, n} & \cdots & b_{k, n}
\end{array}\right) .
$$

By definition, $S_{n, 0}(g, p)=S_{n}(g, p)$.

Theorem Let $g_{1}, g_{2}$, and $g_{3}$ be linearly independent functions in $\mathcal{H}_{n}$. Then for any $0 \leq k<n-1$, the functions

$$
F=\frac{S_{n, k}\left(g_{1}, p_{i}\right)}{S_{n, k}\left(g_{3}, p_{i}\right)}, \quad G=\frac{S_{n, k}\left(g_{2}, p_{i}\right)}{S_{n, k}\left(g_{3}, p_{i}\right)}
$$

satisfy functional equation (25).

We now write the corresponding $(2+1)$-dimensional systems explicitly. According to the lemma in Sec. 4.2, the equation

$$
\sum_{i=1}^{n}\left(A_{i} u_{i, t_{1}}+B_{i} u_{i, t_{2}}+C_{i} u_{i, x}\right)=0
$$


belongs to the system if and only if the expression

$$
\sum_{i=1}^{n} \frac{\lambda_{i}-\lambda_{0}}{p-\lambda_{i}}\left(A_{i} S_{n, k}\left(g_{1}, p\right)+B_{i} S_{n, k}\left(g_{2}, p\right)+C_{i} S_{n, k}\left(g_{3}, p\right)\right)
$$

vanishes identically. We first consider the case $k=0$. Let $g_{i}=a_{i, 0}+a_{i, 1} e^{u_{1}}+$ $\cdots+a_{i, n} e^{u_{n}}, i=1,2,3$. Then the corresponding $(2+1)$-dimensional system is

$$
\begin{aligned}
& \sum_{\substack{j=1, j \neq i}}^{n}\left(a_{2, i} a_{3, j}-a_{2, j} a_{3, i}\right) e^{u_{j}} \frac{u_{i, t_{1}}-u_{j, t_{1}}}{\lambda_{i}-\lambda_{j}}+\left(a_{2, i} a_{3,0}-a_{3, i} a_{2,0}\right) \frac{u_{i, t_{1}}}{\lambda_{i}-\lambda_{0}}+ \\
& \quad+\sum_{\substack{j=1, j \neq i}}^{n}\left(a_{3, i} a_{1, j}-a_{3, j} a_{1, i}\right) e^{u_{j}} \frac{u_{i, t_{2}}-u_{j, t_{2}}}{\lambda_{i}-\lambda_{j}}+\left(a_{3, i} a_{1,0}-a_{1, i} a_{3,0}\right) \frac{u_{i, t_{2}}}{\lambda_{i}-\lambda_{0}}+ \\
& \quad+\sum_{\substack{j=1, j \neq i}}^{n}\left(a_{1, i} a_{2, j}-a_{1, j} a_{2, i}\right) e^{u_{j}} \frac{u_{i, x}-u_{j, x}}{\lambda_{i}-\lambda_{j}}+\left(a_{1, i} a_{2,0}-a_{2, i} a_{1,0}\right) \frac{u_{i, x}}{\lambda_{i}-\lambda_{0}}=0,
\end{aligned}
$$

where $i=1, \ldots, n$. In the simplest case, $n=2$, and we can use linear transformations of the functions $g_{i}$ (see the corollary in Sec. 4.2) to reduce the matrix $a_{i j}$ to the diagonal form $\operatorname{diag}\left(\left(\lambda_{1}-\lambda_{0}\right)^{-1},\left(\lambda_{0}-\lambda_{2}\right)^{-1},\left(\lambda_{2}-\lambda_{1}\right)^{-1}\right)$. System (664) then takes the simple form

$$
u_{y}=e^{v}\left(v_{x}-u_{x}\right), \quad v_{t}=e^{u}\left(u_{x}-v_{x}\right) .
$$

The system in Example7 7 reduces to this system upon some changes of form (11), (10). Introducing the potential

$$
Z_{t}=e^{u}, \quad Z_{y}=-e^{v},
$$

we can write this system as the single equation

$$
\frac{Z_{t y}}{Z_{t} Z_{y}}=\frac{Z_{x y}}{Z_{y}}+\frac{Z_{x t}}{Z_{t}}
$$

If $k>0$, then the corresponding (2+1)-dimensional system contains two 
groups of equations:

$$
\begin{aligned}
& \sum_{\substack{j=1, j \neq i}}^{n-k+1}\left(\Delta_{i}\left(g_{2}\right) \Delta_{j}\left(g_{3}\right)-\Delta_{j}\left(g_{2}\right) \Delta_{i}\left(g_{3}\right)\right) e^{u_{j}} \frac{u_{i, t_{1}}-u_{j, t_{1}}}{\lambda_{i}-\lambda_{j}}+ \\
& +\left(\Delta_{i}\left(g_{2}\right) \Delta_{0}\left(g_{3}\right)-\Delta_{0}\left(g_{2}\right) \Delta_{i}\left(g_{3}\right)\right) \frac{u_{i, t_{1}}}{\lambda_{i}-\lambda_{0}}+ \\
& +\sum_{\substack{j=1, j \neq i}}^{n-k+1}\left(\Delta_{i}\left(g_{3}\right) \Delta_{j}\left(g_{1}\right)-\Delta_{j}\left(g_{3}\right) \Delta_{i}\left(g_{1}\right)\right) e^{u_{j}} \frac{u_{i, t_{2}}-u_{j, t_{2}}}{\lambda_{i}-\lambda_{j}}+ \\
& \left.+\Delta_{i}\left(g_{3}\right) \Delta_{0}\left(g_{1}\right)-\Delta_{0}\left(g_{3}\right) \Delta_{i}\left(g_{1}\right)\right) \frac{u_{i, t_{2}}}{\lambda_{i}-\lambda_{0}}+ \\
& \left.+\sum_{\substack{j=1, j \neq i}}+\left(\Delta_{i}\left(g_{1}\right) \Delta_{j}\left(g_{2}\right)-\Delta_{j}\left(g_{1}\right) \Delta_{i}\left(g_{2}\right)\right) e^{u_{j}} \frac{u_{i, x}-u_{j, x}}{\lambda_{i}-\lambda_{j}}+\Delta_{0}\left(g_{1}\right) \Delta_{i}\left(g_{2}\right)\right) \frac{u_{i, x}}{\lambda_{i}-\lambda_{0}}=0
\end{aligned}
$$

where $i=1, \ldots, n-k$, and

$$
\begin{aligned}
& \sum_{j=1}^{n-k+1} e^{u_{j}} \Delta_{j}\left(g_{r}\right) u_{j, t_{s}}=\sum_{j=1}^{n-k+1} e^{u_{j}} \Delta_{j}\left(g_{s}\right) u_{j, t_{r}}, \\
& \sum_{j=1}^{n-k+1} \Delta_{j}\left(g_{r}\right) e^{u_{j}} \frac{u_{i, t_{s}}-u_{j, t_{s}}}{\lambda_{i}-\lambda_{j}}+\Delta_{0}\left(g_{r}\right) \frac{u_{i, t_{s}}}{\lambda_{i}-\lambda_{0}}=\sum_{j=1}^{n-k+1} \Delta_{j}\left(g_{s}\right) e^{u_{j}} \frac{u_{i, t_{r}}-u_{j, t_{r}}}{\lambda_{i}-\lambda_{j}}+\Delta_{0}\left(g_{s}\right) \frac{u_{i, t_{r}}}{\lambda_{i}-\lambda_{0}},
\end{aligned}
$$

where $i=n-k+2, \ldots, n$. Here, $r, s=1,2,3, t_{3}=x$, and

$$
\begin{aligned}
& \Delta_{j}(g)=\operatorname{det}\left(\begin{array}{ccccc}
g & h_{1} & h_{2} & \cdots & h_{k} \\
a_{j} & b_{1, j} & b_{2, j} & \cdots & b_{k, j} \\
a_{n-k+2} & b_{1, n-k+2} & b_{2, n-k+2} & \cdots & b_{k, n-k+2} \\
\vdots & \vdots & \vdots & \ddots & \vdots \\
a_{n} & b_{1, n} & b_{2, n} & \cdots & b_{k, n}
\end{array}\right), \quad j=1, \ldots, n, \\
& g=a_{0}+a_{1} e^{u_{1}}+\cdots+a_{n} e^{u_{n}}, \\
& h_{1}=b_{1,0}+b_{1,1} e^{u_{1}}+\cdots+b_{1, n} e^{u_{n}},
\end{aligned}
$$

The first group contains $n-k$ equations, and the second group contains $3 k$ equations. There are exactly $k$ linear combinations of equations in the second group that contain no derivatives of the $u_{i}, i=n-k+1, \ldots, n$. These linear combinations can be linearly expressed in terms of the equations in the first group. The 
$(2+1)$-dimensional system therefore contains $(n-k)+3 k-k=n+k$ linearly independent equations.

The simplest nontrivial case is where $n=3$ and $k=1$. In Appendix A we show that the corresponding (2+1)-dimensional system is equivalent to Eq. (7).

\subsection{Degeneracies}

Gibbons-Tsarev system (61) and formulas (63) correspond to the case of pairwise distinct parameters $\lambda_{0}, \ldots, \lambda_{n}$. Here, we consider some reductions of the construction in Sec. 5.1 that correspond to merging these parameters.

We define the polynomials $P_{i}\left(u_{1}, u_{2}, \ldots\right)$ as the coefficients of the Taylor expansion

$$
e^{\varepsilon u_{1}+\varepsilon^{2} u_{2}+\cdots}=1+P_{1} \varepsilon+P_{2} \varepsilon^{2}+\ldots
$$

In particular,

$$
P_{1}\left(u_{1}, u_{2}, \ldots\right)=u_{1}, \quad P_{2}\left(u_{1}, u_{2}, \ldots\right)=u_{2}+\frac{1}{2} u_{1}^{2}, \quad P_{3}\left(u_{1}, u_{2}, \ldots\right)=u_{3}+u_{1} u_{2}+\frac{1}{6} u_{1}^{3} .
$$

We let $Q_{k}\left(\varepsilon, u_{1}, u_{2}, \ldots\right)$ denote the partial sums $1+\sum_{i=1}^{k} P_{i} \varepsilon^{i}$. We set $P_{0}=$ $Q_{0}=1$ by definition.

Let $\lambda_{0}, \ldots, \lambda_{l}$ be pairwise distinct roots with the respective multiplicities $n_{0}+1, n_{1}, \ldots, n_{l}$. Clearly, $n_{0}+\cdots+n_{l}=n$. The corresponding $(n+1)$-field GT system with the field functions $u_{0,1}, \ldots, u_{0, n_{0}}, u_{1,1}, \ldots, u_{1, n_{1}}, \ldots, u_{l, 1}, \ldots, u_{l, n_{l}}, w$ is

$$
\begin{aligned}
& \partial_{i} p_{j}=0, \quad \partial_{i} u_{0, m}=\frac{1}{\left(p_{i}-\lambda_{0}\right)^{m}} \partial_{i} w, \\
& \partial_{i} u_{s, m}=\left(\frac{\lambda_{s}-\lambda_{0}}{\left(p_{i}-\lambda_{s}\right)^{m}}+\frac{1}{\left(p_{i}-\lambda_{s}\right)^{m-1}}\right) \partial_{i} w, \quad \partial_{i} \partial_{j} w=0 .
\end{aligned}
$$

In the case of GT system (65), the solutions $F, G$ of functional equation (25) are given by the formulas in the theorem above where the functions $S_{n, k}$ are defined as follows. We let $\mathcal{H}$ denote the vector space generated by the functions $P_{i}\left(u_{0,1}, u_{0,2}, \ldots\right), i=0, \ldots, n_{0}$, and $e^{u_{s, 1}} P_{i-1}\left(u_{s, 1}, u_{s, 2}, \ldots\right), s=1, \ldots, l, i=$ $1, \ldots, n_{s}$. For any element

$$
g=\sum_{i=0}^{n_{0}} a_{0, i} P_{i}\left(u_{0,1}, u_{0,2}, \ldots\right)+\sum_{s=1}^{l} \sum_{i=1}^{n_{s}} a_{s, i} e^{u_{s, 1}} P_{i-1}\left(u_{s, 1}, u_{s, 2}, \ldots\right)
$$

in this space, we set

$S_{n}(g, p)=\sum_{i=0}^{n_{0}} \frac{a_{0, i} Q_{i}\left(p-\lambda_{0}, u_{0,1}, u_{0,2}, \ldots\right)}{\left(p-\lambda_{0}\right)^{i+1}}+\sum_{s=1}^{l} \sum_{i=1}^{n_{s}} \frac{a_{s, i} e^{u_{s, 1}} Q_{i-1}\left(p-\lambda_{s}, u_{s, 1}, u_{s, 2}, \ldots\right)}{\left(p-\lambda_{s}\right)^{i}}$.

By definition, $S_{n, 0}(g, p)=S_{n}(g, p)$. 
In the case $k>0$, we fix the elements

$h_{j}=\sum_{i=0}^{n_{0}} b_{0, i} P_{i}\left(u_{0,1}, u_{0,2}, \ldots\right)+\sum_{s=1}^{l} \sum_{i=1}^{n_{s}} b_{s, i} e^{u_{s, 1}} P_{i-1}\left(u_{s, 1}, u_{s, 2}, \ldots\right), \quad j=1, \ldots, k$,

in $\mathcal{H}$. Then

$$
S_{n, k}(g, p)=\operatorname{det}\left(\begin{array}{ccccc}
S_{n}(g, p) & S_{n}\left(h_{1}, p\right) & S_{n}\left(h_{2}, p\right) & \ldots & S_{n}\left(h_{k}, p\right) \\
g & h_{1} & h_{2} & \cdots & h_{k} \\
g_{\mathbf{v}_{n-k+2}} & h_{1, \mathbf{v}_{n-k+2}} & h_{2, \mathbf{v}_{n-k+2}} & \cdots & h_{k, \mathbf{v}_{n-k+2}} \\
\vdots & \vdots & \vdots & \ddots & \vdots \\
g_{\mathbf{v}_{n}} & h_{1, \mathbf{v}_{n}} & h_{2, \mathbf{v}_{n}} & \cdots & h_{k, \mathbf{v}_{n}}
\end{array}\right) \text {, }
$$

where $\left(\mathbf{v}_{1}, \ldots, \mathbf{v}_{n}\right)=\left(u_{0,1}, \ldots, u_{0, n_{0}}, u_{1,1}, \ldots, u_{1, n_{1}}, \ldots, u_{l, 1}, \ldots, u_{l, n_{l}}\right)$. We can then easily reconstruct the explicit form of the corresponding (2+1)-dimensional systems using the general construction in the lemma (see Sec. 4.2). We omit this answer for brevity.

\section{Discussion: Classifying integrable $(2+1)$-dimensional systems of hydrodynamic type}

The path from GT systems to integrable $(2+1)$-dimensional systems of hydrodynamic type outlined in Secs. 3 and 4 can be regarded as the following project of classifying integrable (2+1)-dimensional systems.

Step 1 Classify all GT systems (28) (up to equivalence relations (29) and (30)).

Step 2 Classify all the nontrivial solutions $F, G$ of functional equation (25) for each GT system.

Step 3 Use the lemma construction to construct the (2+1)-dimensional systems corresponding to each GT system and each solution of Eq. (25).

In the following remarks, we share our experience acquired during several years of working with integrable systems (11). The major part of these notes are observations and conjectures. We currently have few rigorously proved statements. Most of unproved statements are difficult. In particular, the problem of classifying $n$-field GT systems with arbitrary $n$ is very difficult. But such a classification is apparently possible for lower $n$. We intend to study this problem in the future.

\section{Remarks concerning Step 1}

1. The different general-position GT systems are few and are universal: several families of $(2+1)$-dimensional systems, each family depending on essential parameters, correspond to each of the GT systems. The known GT systems 
correspond to algebraic curves. Apparently, for any genus $g=0,1, \ldots$, we have a unique GT system with one field for $g=0,1$ and $3 g-3$ fields for $g>1$ (see Examples 2 44) such that every integrable general-position (2+1)-dimensional system corresponds to either this GT system or its regular extension. From the algebraic-geometric standpoint, a regular extension corresponds to adding a marked point on the curve.

2. The definition of GT system using formulas (28) admits a coordinate-free reformulation. Let $M$ be a fiber bundle with a one-dimensional fiber $E$ and an $n$-dimensional base $F$. Every $p_{i}$ is then a coordinate on $E$, and $u_{1}, \ldots, u_{n}$ are the coordinates on $F$. We can thus define the notion of the GT structure on $M$. We then apparently have a canonical GT structure on the natural fiber bundle over the moduli space $M_{g}$ of genus- $g$ curves. A fiber $E$ of this fiber bundle is the curve corresponding to a point in $M_{g}$.

3. Integrable systems (11) are defined up to arbitrary pointwise transformations $\mathbf{u} \rightarrow \Phi(\mathbf{u})$. The equivalence problem for systems (1) is nontrivial; in particular, finding a coordinate system in which a $(2+1)$-dimensional system has the simplest form is often difficult. One possible approach to this problem is based on the observation that the simplest coordinates for a GT system, as a rule, are simultaneously the simplest ones for the corresponding $(2+1)$ dimensional system.

4. "Proper" GT system coordinates admit an algebraic-geometric interpretation. For example, we can interpret each of the variables $p_{i}$ and $u$ in Example2 as a coordinate on $\mathbb{C} P^{1}$ and each of the variables $p_{i}$ in Example 3 as a coordinate on an elliptic curve with $\tau$ being the modular parameter of this curve (i.e., a coordinate on the moduli space of genus-one curves). The field functions $u, v$, and $w$ in Example 4 are coordinates on the moduli space of genus-two curves. We expect that in the case $g>2$, we have a GT system whose $3 g-3$ field functions are coordinates on the moduli space $M_{g}$ of genus- $g$ curves. Finding good formulas here is hindered by the absence of a good choice of coordinates on $M_{g}$. We can obtain an algebraic-geometric description of these GT systems simultaneously with the description of hydrodynamic reductions of the $(2+1)$ dimensional systems from Krichever's paper [6]. As soon as we find GT systems related to curves of genus $g>2$, describing solutions of the corresponding functional equation (25) would apparently result in constructing (2+1)-dimensional systems depending on essential parameters. We must then obtain the first examples of generalized hypergeometric functions related to higher-genus curves. This program was realized in [20, 28, for $g=0,1$. We note that the $(2+1)$ dimensional systems in [6] do not contain essential parameters and correspond to the simplest integer values of parameters in the hypergeometric series.

5. The problem of describing all the GT systems with a given number of fields includes the problem of describing degenerations of a general position system. Degenerations appearing when merging the field functions $u_{i}$ (merging the marked points in the algebraic-geometric language) can be described using Young diagrams. In Sec. 3.2, we considered the degeneration of the GT system in Example 2 under which all the points merge into a single point. Gibbons- 
Tsarev system (61) corresponds to degeneration of the type $u_{i} \rightarrow \lambda_{i}+\varepsilon u_{i}$, $\varepsilon \rightarrow 0$.

\section{Remarks concerning Step 2}

6. Algebraic curves in the classification of integrable $(2+1)$-dimensional systems appear in Step 2. In all the known examples, solutions $F, G$ of functional equation (25) are algebraically dependent as functions of $p$. The polynomial relation between them is called the dispersion curve (see (22)). If we are interested in solutions $F, G$ parameterizing a curve of a given genus for a given GT system, then we assume that the dependence of $F, G$ on $p$ is known. For example, in the case of a rational curve, the functions $F, G$ are rational functions of $p$. Different choices of the degrees of $F, G$ result in different families of $(2+1)$ dimensional systems. The functional equation becomes a system of nonlinear differential equations for the ansatz coefficients after the corresponding ansatz is substituted.

7. If we additionally assume that system (11) admits a pseudopotential representation, then we can find solutions $F, G$ using functional equation (76), which is simpler than (25) (see Appendix B).

8. The class of integrable systems (11) is closed under changes of variables (11). The existence of this group leads to formulas (50) for solutions $F, G$ of functional equation (25). In these formulas, the expression for $S(h, p)$ is linear in $h$, and $h_{1}, h_{2}$, and $h_{3}$ are arbitrary elements of a vector space $\mathcal{H}$ of dimension $M$. In the general position case, $\mathcal{H}$ is the solution space for an overdetermined system of linear partial differential equations of hypergeometric type. We can interpret formulas (50) as a linearizing substitution for the nonlinear system mentioned in item 5 . The space $\mathcal{H}$ may consist of elementary functions in the case of reduction. For example, the space $\mathcal{H}$ consists of quasipolynomials in Sec. 5

9. In the case of curves of genus $g=0,1$, the dimension of $\mathcal{H}$ is $n+1$, where $n$ is the number of marked points. The set of times $t_{i}, i=1, \ldots, n+1$, in $\mathcal{H}$ corresponds to the basis $h_{i}, i=1, \ldots, n+1$. For any triple $t_{i}, t_{j}, t_{k}$ of times, formula (50) results in a three-dimensional system of form (11) with the independent variables $t_{i}, t_{j}$, and $t_{k}$. All the systems corresponding to different triples of times are mutually compatible.

\section{A Conservation laws of hydrodynamic type and integrable quasilinear second-order equations}

A relation of the form

$$
a(\mathbf{u})_{t}+b(\mathbf{u})_{y}+c(\mathbf{u})_{x}=0,
$$

where $a, b$, and $c$ are some scalar functions, that is satisfied by any solution $\mathbf{u}$ of system (55) is called a conservation law of hydrodynamic type for system (11). All known integrable systems (10) have a rich collection of conservation laws. 
More precisely, we consider systems (11) with $k=0$. It was proved in [20] that systems (55) corresponding to a rational nonsingular curve (Example 1) have $n+1$ linearly independent conservation laws; all partial derivatives of the functions $a, b$, and $c$ with respect to the $u_{i}$ were found explicitly there. The dimension of the space of conservation laws is apparently $n$ in the case of an elliptic curve (Example 3). This was verified for degenerations of the elliptic systems presented in [7].

In the case of exponential systems (64) with $k=0$, we can find conservation laws explicitly.

Proposition 4 System (64) with $k=0$ admits the $n+1$ conservation laws $\left|\begin{array}{cc}S_{n}^{\mathrm{reg}}\left(g_{2}, \lambda_{i}\right) & S_{n}^{\mathrm{reg}}\left(g_{3}, \lambda_{i}\right) \\ a_{2, i} e^{-u_{i}} & a_{3, i} e^{-u_{i}}\end{array}\right|_{t}\left|\begin{array}{cc}S_{n}^{\mathrm{reg}}\left(g_{3}, \lambda_{i}\right) & S_{n}^{\mathrm{reg}}\left(g_{1}, \lambda_{i}\right) \\ a_{3, i} e^{-u_{i}} & a_{1, i} e^{-u_{i}}\end{array}\right|_{y}+\left|\begin{array}{cc}S_{n}^{\mathrm{reg}}\left(g_{1}, \lambda_{i}\right) & S_{n}^{\mathrm{reg}}\left(g_{2}, \lambda_{i}\right) \\ a_{1, i} e^{-u_{i}} & a_{2, i} e^{-u_{i}}\end{array}\right|_{x}=0$,

where $i=1, \ldots, n$, and $\left|\begin{array}{cc}S_{n}^{\mathrm{reg}}\left(g_{2}, \lambda_{0}\right) & S_{n}^{\mathrm{reg}}\left(g_{3}, \lambda_{0}\right) \\ a_{2,0} & a_{3,0}\end{array}\right|_{t}\left|\begin{array}{cc}S_{n}^{\mathrm{reg}}\left(g_{3}, \lambda_{0}\right) & S_{n}^{\mathrm{reg}}\left(g_{1}, \lambda_{0}\right) \\ a_{3,0} & a_{1,0}\end{array}\right|_{y}+\left|\begin{array}{cc}S_{n}^{\mathrm{reg}}\left(g_{1}, \lambda_{0}\right) & S_{n}^{\mathrm{reg}}\left(g_{2}, \lambda_{0}\right) \\ a_{1,0} & a_{2,0}\end{array}\right|_{x}=0$.

Here,

$S_{n}^{\mathrm{reg}}\left(g, \lambda_{i}\right)=\left.\left(S_{n}(g, p)-\frac{a_{i} e^{u_{i}}}{p-\lambda_{i}}\right)\right|_{p=\lambda_{i}}, \quad S_{n}^{\mathrm{reg}}\left(g, \lambda_{0}\right)=\left.\left(S_{n}(g, p)-\frac{a_{0}}{p-\lambda_{0}}\right)\right|_{p=\lambda_{0}}$.

In the case $k>0$, all known integrable systems (1) have $k$ triples of "short" conservation laws of the form

$$
a_{y}=b_{x}, \quad a_{t}=c_{x}, \quad b_{t}=c_{y} .
$$

We can introduce the potential $Z_{x}=a, Z_{y}=b$, and $Z_{t}=c$ for each such triple. For small $k$ (for $n \geq 2 k$ in the rational case and for $n \geq 2 k-1$ in the elliptic case), introducing potentials $Z_{1}, \ldots, Z_{k}$, we make system (11) a properly defined system consisting of several second-order equations and several firstorder equations. In particular, for $n=3 k$, system (11) is equivalent to a system of the form

$$
A_{1} \mathbf{Z}_{t t}+A_{2} \mathbf{Z}_{y t}+A_{3} \mathbf{Z}_{x t}+A_{4} \mathbf{Z}_{y y}+A_{5} \mathbf{Z}_{x y}+A_{6} \mathbf{Z}_{x x}=0
$$

where $\mathbf{Z}=\left(Z_{1}, \ldots, Z_{k}\right)^{\mathrm{T}}$ and $A_{i}$ are some $k \times k$ matrices depending on $\mathbf{Z}_{x}, \mathbf{Z}_{y}$, and $\mathbf{Z}_{t}$.

Short conservation laws for the systems in Sec. 4.1 with $k>0$ are given by the formulas

$$
\left(\frac{\Delta\left(g_{r}, h_{1}, \ldots, \hat{i}, \ldots, h_{k}\right)}{\Delta\left(h_{1}, \ldots, h_{k}\right)}\right)_{t_{s}}=\left(\frac{\Delta\left(g_{s}, h_{1}, \ldots, \hat{i}, \ldots, h_{k}\right)}{\Delta\left(h_{1}, \ldots, h_{k}\right)}\right)_{t_{r}},
$$


where $i=1, \ldots, k, r, s=1,2,3, t_{1}=t, t_{2}=y, t_{3}=x$, and

$$
\Delta\left(f_{1}, \ldots, f_{k}\right)=\operatorname{det}\left(\begin{array}{cccc}
f_{1} & f_{2} & \cdots & f_{k} \\
f_{1, u_{n-k+2}} & f_{2, u_{n-k+2}} & \cdots & f_{k, u_{n-k+2}} \\
\vdots & \vdots & \ddots & \vdots \\
f_{1, u_{n}} & f_{2, u_{n}} & \cdots & f_{k, u_{n}}
\end{array}\right) .
$$

In addition to these conservation laws, such systems apparently also have $n+1$ linearly independent conservation laws of hydrodynamic type.

In the case where $n=3$ and $k=1$, the system in Sec. 4.1 depends on four arbitrary linearly independent constant vectors (see (51)). After the potential is introduced using the short conservation laws, the system becomes an equation of form (2) whose coefficients $A_{i}$ are polynomials of a degree not exceeding two in the variables $Z_{x}, Z_{y}$, and $Z_{t}$. Using the group $G L(4)$ acting on the set of equations (21), we can reduce the matrix composed from the above vectors to the unit matrix by linear transformations of the variables $Z, x, t$, and $y$. The most convenient way to do this is to make the function $h$ equal to unity. Then, in the general position case where $n_{0}=0$ and $n_{1}=n_{2}=n_{3}=1$ (see (65)), the equation coincides with (7). The degeneration of the form $n_{0}=3$ and $n_{1}=n_{2}=n_{3}=0$ results in the equation [4]

$$
Z_{x t}-Z_{y y}+Z_{x} Z_{x y}-Z_{y} Z_{x x}=0,
$$

the degeneration $n_{0}=2, n_{1}=1$, and $n_{2}=n_{3}=0$ gives

$$
Z_{y t}+Z_{t} Z_{x x}-Z_{x} Z_{x t}=0,
$$

the degeneration $n_{0}=1, n_{2}=2$, and $n_{2}=n_{3}=0$ gives

$$
Z_{y} Z_{x t}-Z_{t} Z_{x y}+Z_{y y}=0,
$$

and the degeneration $n_{0}=n_{1}=n_{2}=1$ and $n_{3}=0$ gives

$$
Z_{y} Z_{x t}-Z_{t} Z_{x y}+Z_{y t}=0 .
$$

\section{B The GT systems and the pseudopotential rep- resentation}

In Sec. 4. we described several classes of solutions of functional equation (25) for the rational and elliptic GT systems. These solutions were found in 20, 28, under the additional assumption that in addition to hydrodynamic reductions, the corresponding (2+1)-dimensional systems (1) have the pseudopotential representation or, equivalently, the dispersionless Lax representation. As we demonstrate below, this assumption results in a functional equation that is simpler than (25) and was in fact solved. 
The dispersionless Lax representation is the relation

$$
L_{t}=\{L, A\},
$$

where $\{L, A\}=A_{\lambda} L_{x}-A_{x} L_{\lambda}$. Here, $A=A\left(\lambda, u_{1}(x, t), \ldots, u_{n}(x, t)\right)$ and the unknown function $L=L\left(\lambda, u_{1}(x, t), \ldots, u_{n}(x, t)\right)$ depend on the spectral parameter $\lambda$. The transformation $L(x, t, \lambda) \rightarrow \lambda(x, t, L)$ takes (69) to the conservative form

$$
\lambda_{t}=A\left(\lambda, u_{1}, \ldots, u_{n}\right)_{x}
$$

where $L$ now plays the role of a hidden parameter. We can rewrite the last equation as

$$
\Phi_{t}=A\left(\Phi_{x}, u_{1}, \ldots, u_{n}\right),
$$

where $\lambda=\Phi_{x}$.

We assume that system (1) has both hydrodynamic reductions (24) and pseudopotential representation (4). Calculating $L_{t}$ and $L_{x}$ on the $N$-phase solutions and using (43), we write (69) as

$\sum_{i} F\left(p_{i}, u_{1}, \ldots, u_{n}\right) \partial_{i} L r_{x}^{i}=\sum_{i}\left(\partial_{i} L A_{\lambda}\left(\lambda, u_{1}, \ldots, u_{n}\right)-L_{\lambda} \sum_{k=1}^{n} A_{u_{k}}\left(\lambda, u_{1}, \ldots, u_{n}\right) \partial_{i} u_{k}\right) r_{x}^{i}$.

Equating the coefficients of $r_{x}^{i}$, we obtain

$$
F\left(p_{i}, u_{1}, \ldots, u_{n}\right) \partial_{i} L=A_{\lambda}\left(\lambda, u_{1}, \ldots, u_{n}\right) \partial_{i} L-L_{\lambda} \sum_{k=1}^{n} A_{u_{k}}\left(\lambda, u_{1}, \ldots, u_{n}\right) \partial_{i} u_{k} .
$$

The function $L$ therefore satisfies the system of equations

$$
\partial_{i} L=t\left(p_{i}, \lambda, u_{1}, \ldots, u_{n}\right) L_{\lambda} \partial_{i} u_{1}, \quad i=1, \ldots, N,
$$

which is compatible by virtue of the corresponding GT system (28). System (72) is called the Löwner equation.

Using transformation (29), we can arbitrarily gauge the function $F$. For example, we can set $F=p_{i}$. It is convenient to choose

$$
F\left(p_{i}, u_{1}, \ldots, u_{n}\right)=A_{p_{i}}\left(p_{i}, u_{1}, \ldots, u_{n}\right),
$$

and system (72) then becomes

$$
\partial_{i} L=\frac{\sum_{k=1}^{n} A_{u_{k}}\left(\lambda, u_{1}, \ldots, u_{n}\right) \partial_{i} u_{k}}{A_{\lambda}\left(\lambda, u_{1}, \ldots, u_{n}\right)-A_{p_{i}}\left(p_{i}, u_{1}, \ldots, u_{n}\right)} L_{\lambda}, \quad i=1, \ldots, N .
$$

The transformation $L(x, t, \lambda) \rightarrow \lambda(x, t, L)$ brings (73) to the form

$$
\partial_{i} \lambda=\frac{\sum_{k=1}^{n} A_{u_{k}}\left(\lambda, u_{1}, \ldots, u_{n}\right) \partial_{i} u_{k}}{A_{p_{i}}\left(p_{i}, u_{1}, \ldots, u_{n}\right)-A_{\lambda}\left(\lambda, u_{1}, \ldots, u_{n}\right)}, \quad i=1, \ldots, N .
$$


Relation (73) implies that $L_{\lambda}=0$ at the points $\lambda=p_{i}, i=1, \ldots, N$. Substituting $\lambda=p_{i}$ in (69), we obtain

$$
\left(L\left(p_{i}, r^{1}, \ldots, r^{N}\right)\right)_{t}=A_{p_{i}}\left(p_{i}, u_{1}, \ldots, u_{n}\right)\left(L\left(p_{i}, r^{1}, \ldots, r^{N}\right)\right)_{x} .
$$

We see that the functions $L\left(p_{i}, r^{1}, \ldots, r^{N}\right)$ and $r_{i}$ satisfy system (43) with the same functions $F_{i}$. Hence, $L\left(p_{i}, r^{1}, \ldots, r^{N}\right)=\mu_{i}\left(r^{i}\right)$ for some functions $\mu_{i}$. After changing the Riemann invariants $\mu_{i}\left(r^{i}\right) \rightarrow r^{i}$, we can set

$$
r^{i}=L\left(p_{i}, r^{1}, \ldots, r^{N}\right)
$$

After the transformation $L(x, t, \lambda) \rightarrow \lambda(x, t, L)$, this relation becomes

$$
p_{i}=\lambda\left(x, t, r^{i}\right)
$$

Substituting $L=p_{j}$ in (74), we obtain the relation

$$
\partial_{i} p_{j}=\frac{\sum_{k=1}^{n} A_{u_{k}}\left(p_{j}, u_{1}, \ldots, u_{n}\right) \partial_{i} u_{k}}{A_{p_{i}}\left(p_{i}, u_{1}, \ldots, u_{n}\right)-A_{p_{j}}\left(p_{j}, u_{1}, \ldots, u_{n}\right)}, \quad i \neq j, \quad i, j=1, \ldots, N
$$

which expresses the function $f\left(p_{i}, p_{j}, u_{1}, \ldots, u_{n}\right)$ in the corresponding GT system (28) in terms of the potential $A$ and the functions $g_{2}, \ldots, g_{n}$. This formula for $n=1$ is contained in 42 .

We recall that the GT-system gauge is fixed by the condition $F=A_{p_{i}}$ in formula (75). This gauge is generally inconvenient. After an arbitrary transformation (29), formula (75) becomes

$\partial_{i} p_{j}=\frac{\sum_{k=1}^{n}\left(\Phi_{p_{i}}\left(p_{i}, u_{1}, \ldots, u_{n}\right) \phi_{u_{k}}\left(p_{j}, u_{1}, \ldots, u_{n}\right)-\phi_{p_{i}}\left(p_{i}, u_{1}, \ldots, u_{n}\right) \Phi_{u_{k}}\left(p_{j}, u_{1}, \ldots, u_{n}\right)\right) \partial_{i} u_{k}}{\phi_{p_{i}}\left(p_{i}, u_{1}, \ldots, u_{n}\right) \Phi_{p_{j}}\left(p_{j}, u_{1}, \ldots, u_{n}\right)-\phi_{p_{j}}\left(p_{j}, u_{1}, \ldots, u_{n}\right) \Phi_{p_{i}}\left(p_{i}, u_{1}, \ldots, u_{n}\right)}$,

where $i \neq j, i, j=1, \ldots, N$, and the potential is defined parametrically

$$
A=\phi\left(p, u_{1}, \ldots, u_{n}\right), \quad \lambda=\Phi\left(p, u_{1}, \ldots, u_{n}\right) .
$$

Using (28) and omitting the arguments $u_{1}, \ldots, u_{n}$ for brevity, we rewrite formula (76) in the form

$$
f\left(p_{i}, p_{j}\right)=\frac{\sum_{k=1}^{n}\left(\Phi_{p_{i}}\left(p_{i}\right) \phi_{u_{k}}\left(p_{j}\right)-\phi_{p_{i}}\left(p_{i}\right) \Phi_{u_{k}}\left(p_{j}\right)\right) g_{k}\left(p_{i}\right)}{\phi_{p_{i}}\left(p_{i}\right) \Phi_{p_{j}}\left(p_{j}\right)-\phi_{p_{j}}\left(p_{j}\right) \Phi_{p_{i}}\left(p_{i}\right)}
$$

where $g_{1}=1$. For a fixed GT system (28), this relation is a functional equation for the functions $\phi$ and $\Phi$ that is much simpler than functional equation (25) for the functions $F$ and $G$. In particular, fixing $p_{j}$, we can find the dependence of the ratio $\phi_{p_{i}} / \Phi_{p_{i}}$ on the variable $p_{i}$ from (77). Every solution of Eq. (77) determines an integrable potential corresponding to system (28).

Example 2a (continuation of Example 2). We find the one-field integrable potentials corresponding to one-field GT system (34) with $P(x)=x(x-1)$. 
Fixing $p_{j}$ in (77), we find that $\phi_{p_{i}} / \Phi_{p_{i}}$ is the ratio of polynomials of the first degree in $p_{i}$. We set

$$
\Phi_{p_{i}}=\left(A_{1}(u) p_{i}+A_{0}(u)\right) Z\left(p_{i}, u\right), \quad \phi_{p_{i}}=\left(B_{1}(u) p_{i}+B_{0}(u)\right) Z\left(p_{i}, u\right) .
$$

Substituting these expressions in (77) and equating the coefficients of like powers of $p_{j}$, we find that (77) is equivalent to the relations

$$
\begin{aligned}
& \Phi_{u}=-\left(A_{1}(u) u+A_{0}(u)\right) \frac{p_{i}\left(p_{i}-1\right)}{u(u-1)} Z\left(p_{i}, u\right), \\
& \phi_{u}=-\left(B_{1}(u) u+B_{0}(u)\right) \frac{p_{i}\left(p_{i}-1\right)}{u(u-1)} Z\left(p_{i}, u\right) .
\end{aligned}
$$

We find both partial derivatives of the function $Z\left(p_{i}, u\right)$ from the compatibility condition for these two systems (i.e., from the condition that the mixed derivatives of $\phi$ and $\Phi$ are equal). We then find the condition for the equality of the mixed derivatives of $Z$. Equating the coefficients of like powers of $p_{i}$ in this relation, we obtain a system of nonlinear ODEs for the functions $A_{i}$ and $B_{i}$, $i=0,1$. We can express a solution of this system in terms of two arbitrary solutions $y_{1}$ and $y_{2}$ of the standard hypergeometric equation

$$
u(u-1) y(u)^{\prime \prime}+[(\alpha+\beta+1) u-\gamma] y(u)^{\prime}+\alpha \beta y(u)=0
$$

as

$$
A_{1}=-\alpha y_{1}, \quad A_{0}=u(u-1) y_{1}^{\prime}+\alpha u y_{1}, \quad B_{1}=-\alpha y_{2}, \quad B_{0}=u(u-1) y_{2}^{\prime}+\alpha u y_{2} .
$$

We reconstruct the function $Z$ from its partial derivatives:

$$
Z(p, u)=(p-1)^{\alpha+\gamma} p^{-\beta-\gamma-1}(p-u)^{\beta-1} .
$$

We finally obtain

$$
\Phi(p, u)=\int_{0}^{p}\left[u(u-1) y_{1}^{\prime}(u)+\alpha(u-\xi) y_{1}(u)\right](\xi-1)^{\alpha+\gamma} \xi^{-\beta-\gamma-1}(\xi-u)^{\beta-1} d \xi .
$$

To obtain the function $\phi$, we merely substitute $y_{2}$ for $y_{1}$ in this formula.

The potential found above corresponds to the general position case in 27, where all the integrable one-field potentials were described. The generalization of formula (71) to the case of $n$-field GT system (45), (46) is

$P_{n}(p, h)=\int_{0}^{p} S_{n}(h, \xi)\left(\xi-u_{1}\right)^{-s_{1}-1} \cdots\left(\xi-u_{n}\right)^{-s_{n}-1} \xi^{-s_{n+1}-1}(\xi-1)^{-s_{n+2}-1} d \xi$,

where $h\left(u_{1}, \ldots, u_{n}\right)$ is an arbitrary solution of system (47), (48) and the polynomial $S_{n}$ is given by formula (49). The elliptic analogue of this potential is given by

$$
P_{n}(p, h)=\int_{0}^{p} S_{n}(h, \xi) e^{2 \pi i r(\tau-\xi)} \frac{\theta^{\prime}(0)^{-s_{1}-\cdots-s_{n}} \theta\left(u_{1}\right)^{s_{1}} \cdots \theta\left(u_{n}\right)^{s_{n}}}{\theta(\xi)^{-s_{1}-\cdots-s_{n}} \theta\left(\xi-u_{1}\right)^{s_{1}} \cdots \theta\left(\xi-u_{n}\right)^{s_{n}}} d \xi,
$$


where we use the notation in Example 3. Integrable potentials with the defect index $k>0$ corresponding to rational and elliptic curves were presented in [20], [28].

Clearly, for any solution $\phi, \Phi$ of Eq. (77), the pair $k_{1} \phi+k_{2} \Phi, k_{3} \phi+k_{4} \Phi$ is a solution for any constant $k_{i}$. In the above example, such a transformation corresponds to another choice of the hypergeometric equation solutions $y_{1}, y_{2}$. The function $\phi$ therefore always lies in a two-dimensional space $V$. If this space has the dimension $d \geq 3$, then choosing linearly independent elements $\phi_{1}, \phi_{2}, \phi_{3} \in V$, we obtain the functions

$$
F=\frac{\left(\phi_{1}\right)_{p_{i}}}{\left(\phi_{3}\right)_{p_{i}}}, \quad G=\frac{\left(\phi_{2}\right)_{p_{i}}}{\left(\phi_{3}\right)_{p_{i}}}
$$

satisfying functional equation (25). This is how solutions of Eq. (25) were found in [20], 28]. Equation (25) for GT system (61) was solved directly.

\section{Multidimensional integrable systems of hy- drodynamic type}

Multidimensional generalizations of systems (11) are systems of the form

$$
\sum_{i=1}^{d} A_{i}(\mathbf{u}) \frac{\partial \mathbf{u}}{\partial x_{i}}=0, \quad d>3
$$

where $A_{i}$ are $n \times n$ matrices and $\mathbf{u}=\left(u_{1}, \ldots, u_{n}\right)$. We do not here discuss the definition of the integrability of such systems based on the hydrodynamic reduction method (see [15]). But if we know what integrable system (11) is, we can naturally require that every reduction

$$
\mathbf{u}=\mathbf{u}\left(\sum_{i=1}^{d} k_{1, i} x_{i}, \sum_{i=1}^{d} k_{2, i} x_{i}, \sum_{i=1}^{d} k_{3, i} x_{i}\right)
$$

of integrable system (79) result in a three-dimensional integrable system (1) for any constants $k_{i, j}$. We note that the coefficients of this system of form (11) depend on the parameters $k_{i, j}$ linearly. It was shown in [43] that in the case $n=2$, systems (79) have this property, being reductions of the system

$$
u_{x_{1}}+v_{x_{2}}+u v_{x_{3}}-v u_{x_{3}}=0, \quad u_{x_{4}}+v_{x_{5}}+u v_{x_{6}}-v u_{x_{6}}=0 .
$$

System (81) admits a maximum collection of hydrodynamic reductions and can be written as the commutation condition for a pair of vector fields. This system was first derived in [15] as follows. We have four parameters (namely, the coefficients $p_{0}, p_{1}, p_{2}$, and $p_{3}$ of the polynomial $P$ ) in system (58) entering the system coefficients linearly. If we write (58) as

$$
\mathbf{u}_{t}+A(\mathbf{u}) \mathbf{u}_{x}+\sum_{i=0}^{3} p_{i} B_{i}(\mathbf{u}) \mathbf{u}_{y}=0
$$


and pass to the six-dimensional system

$$
\mathbf{u}_{t}+A(\mathbf{u}) \mathbf{u}_{x}+\sum_{i=0}^{3} B_{i}(\mathbf{u}) \mathbf{u}_{y_{i}}=0
$$

then this last system is equivalent to system (81).

Analyzing system (58) and other known examples of multidimensional integrable dispersionless systems, we proposed the conjecture that for any system (1) obtained by reduction (80) from an integrable multidimensional system, the corresponding GT system is equivalent to system (37) [44.

In Sec. 5, we constructed a broad class of systems (11) whose corresponding GT systems have form (37). Systems (64) are the simplest ones. Below, we attempt to construct multidimensional integrable systems corresponding to these systems.

System (64) is written in the coordinates $u_{i}$ in which the corresponding GT system acquires the simplest form. According to Proposition 3, system (64) admits a pseudopotential representation depending rationally on the spectral parameter. Taking zeros of the pseudopotential representation denominator as the new field functions $v_{i}, i=1, \ldots, n$, we reduce the system to the form analogous to (58)

$$
\left(v_{i}\right)_{t}-\frac{f_{v_{i}}}{g_{v_{i}}}\left(v_{i}\right)_{x}+\left(\sum_{k \neq i} \gamma_{i k} P\left(v_{k}\right)+\delta_{i}\right)\left(v_{i}\right)_{y}+\sum_{j \neq i} \beta_{i j} P\left(v_{i}\right)\left(v_{j}\right)_{y}=0, \quad i=1, \ldots, n,
$$

where $\beta_{i j}, \gamma_{i k}$, and $\delta_{i}$ are expressed in terms of the two symmetric polynomials

$$
\begin{aligned}
& f(\mathbf{v})=f_{0}+f_{1}\left(v_{1}+\cdots+v_{n}\right)+\cdots+f_{n} v_{1} \cdots v_{n}, \\
& g(\mathbf{v})=g_{0}+g_{1}\left(v_{1}+\cdots+v_{n}\right)+\cdots+g_{n} v_{1} \cdots v_{n}
\end{aligned}
$$

(which are linear in each of the variables) and the polynomial $P(x)=x^{n+1}+$ $p_{n} x^{n}+\cdots+p_{0}$ as

$$
\begin{aligned}
& \beta_{i j}=\frac{f_{v_{i} v_{j}} g_{v_{i}}-g_{v_{i} v_{j}} f_{v_{i}}}{\left(v_{i}-v_{1}\right) \cdots\left(v_{i}-v_{n}\right) g_{v_{i}}}, \quad \gamma_{i k}=\frac{f_{v_{i}} g_{v_{k}}-g_{v_{i}} f_{v_{k}}}{\left(v_{i}-v_{1}\right) \cdots\left(v_{i}-v_{n}\right) g_{v_{i}}\left(v_{i}-v_{k}\right)}, \\
& \delta_{i}=\frac{g f_{v_{i}}-f g_{v_{i}}}{g_{v_{i}}} .
\end{aligned}
$$

Conjecture 1 The corresponding $(n+4)$-dimensional system

$$
\left(v_{i}\right)_{t}-\frac{f_{v_{i}}}{g_{v_{i}}}\left(v_{i}\right)_{x}+\sum_{\substack{m=0, k \neq i}}^{n+1} \gamma_{i k} v_{k}^{m}\left(v_{i}\right)_{y_{m}}+\delta_{i}\left(v_{i}\right)_{y_{n+1}}+\sum_{\substack{m=0, j \neq i}}^{n+1} \beta_{i j} v_{i}^{m}\left(v_{j}\right)_{y_{m}}=0,
$$

$i=1, \ldots, n$, is integrable for any $n$. 
We note that for $n=3$, we can easily reduce system (82) to the form analogous to (81)

$$
\begin{aligned}
& u_{x_{1}}-w_{x_{2}}+u w_{x_{3}}-w u_{x_{3}}=0, \quad v_{y_{1}}-w_{y_{2}}+v w_{y_{3}}-w v_{y_{3}}=0 \\
& w_{z}=v_{x_{1}}-u_{y_{1}}+v w_{x_{3}}-w v_{x_{3}}-u w_{y_{3}}+w u_{y_{3}} .
\end{aligned}
$$

We plan to reduce system (82) to a simpler form for arbitrary $n$, to prove that it is integrable, and to study the corresponding GT system in a separate paper. Here, we only note that the definition of the GT system as a compatible system of form (28) can be easily generalized to the case $d>3$. Moreover, if GT systems in the three-dimensional case are related to fiber bundles with a one-dimensional fiber (see Sec.6), then a fiber in the $d$-dimensional case has the dimension $d-2$. Some GT systems for $d>3$ were found in [15. We present a few new examples of one-field GT systems in the case $d=4$.

Let $P(x)=z_{2} x^{2}+z_{1} x+z_{0}$ and $Q(x)$ be two arbitrary quadratic polynomials, $J(x)$ be an arbitrary linear polynomial, and $S(x, y)=2 z_{2} x y+z_{1}(x+y)+2 z_{0}$.

Example 8 The formulas

$$
\begin{aligned}
& \partial_{i} p_{j}=\frac{\left(p_{i}-p_{j}\right)^{2}}{P\left(p_{i}\right)\left(q_{i}-q_{j}\right)} \partial_{i} u, \quad \partial_{i} q_{j}=\frac{Q\left(p_{i}\right)}{P\left(p_{i}\right)} \partial_{i} u \\
& \partial_{i} \partial_{j} u=\frac{\left(p_{i}-p_{j}\right) S\left(p_{i}, p_{j}\right)}{P\left(p_{i}\right) P\left(p_{j}\right)\left(q_{i}-q_{j}\right)} \partial_{i} u \partial_{j} u
\end{aligned}
$$

give a one-field GT system with a two-dimensional fiber. The coordinates in the fiber are $p_{i}$ and $q_{i}$, and $u$ is the coordinate on the base. Using a fractional-linear transformation of $p$, we can reduce the polynomial $P$ to one of the two canonical forms $P(x)=x$ or $P(x)=1$. The case $P(x)=1$ in other coordinates arose in [15] when investigating the equation

$$
Z_{t x}+Z_{x y}+Z_{x x} Z_{y y}-Z_{x y}^{2}=0 .
$$

The general position case $P(x)=x$ is possibly related to the most nondegenerate integrable equation of this sort.

Example 9 The formulas

$$
\begin{aligned}
\partial_{i} p_{j} & =\frac{\left(p_{i}-p_{j}\right) J\left(p_{i}\right) J\left(p_{j}\right)}{P\left(p_{i}\right)\left(q_{i}-q_{j}\right)^{2}} \partial_{i} u, \quad \partial_{i} q_{j}=\frac{J\left(p_{i}\right)^{2}}{P\left(p_{i}\right)\left(q_{i}-q_{j}\right)} \partial_{i} u, \\
\partial_{i} \partial_{j} u & =\frac{J\left(p_{i}\right) J\left(p_{j}\right) S\left(p_{i}, p_{j}\right)}{P\left(p_{i}\right) P\left(p_{j}\right)\left(q_{i}-q_{j}\right)^{2}} \partial_{i} u \partial_{j} u
\end{aligned}
$$

give a one-field GT system. 
Example 10 The formulas

$$
\begin{aligned}
& \partial_{i} p_{j}=\frac{p_{i} p_{j}\left(2-p_{j}\right)}{\left(q_{i}-q_{j}\right)^{2}} \partial_{i} u, \quad \partial_{i} q_{j}=\frac{p_{i}}{q_{i}-q_{j}} \partial_{i} u, \\
& \partial_{i} \partial_{j} u=\frac{p_{i} p_{j}}{\left(q_{i}-q_{j}\right)^{2}} \partial_{i} u \partial_{j} u
\end{aligned}
$$

give a one-field GT system.

We do not know whether the GT systems in Examples 9 and 10 are related to some multidimensional integrable dispersionless systems.

\section{Classifying integrable chains of hydrodynamic type}

We consider integrable quasilinear infinite chains of the form

$$
u_{\alpha, t}=\phi_{\alpha, 1} u_{1, x}+\cdots+\phi_{\alpha, \alpha+1} u_{\alpha+1, x}, \quad \alpha=1,2, \ldots, \quad \phi_{\alpha, \alpha+1} \neq 0,
$$

where $\phi_{\alpha, j}=\phi_{\alpha, j}\left(u_{1}, \ldots, u_{\alpha+1}\right)$. Two chains are equivalent if they are related by a transformation of the form

$$
u_{\alpha} \rightarrow \Psi_{\alpha}\left(u_{1}, \ldots, u_{\alpha}\right), \quad \frac{\partial \Psi_{\alpha}}{\partial u_{\alpha}} \neq 0, \quad \alpha=1,2, \ldots
$$

We consider a chain integrable if it admits hydrodynamic reductions (see [13[15], 23]-25]).

Example 11 The Benney chain [45]-47]

$u_{1, t}=u_{2, x}, \quad u_{2, t}=u_{1} u_{1, x}+u_{3, x}, \quad \ldots, \quad u_{\alpha t}=(\alpha-1) u_{\alpha-1} u_{1, x}+u_{\alpha+1, x}$,

is the best-known example of an integrable chain (83). Hydrodynamic reductions of the Benney chain were studied in [12].

Definition 9 A hydrodynamic (1+1)-dimensional $N$-component reduction of chain (83) is semi-Hamiltonian system (43) in which the functions $u_{j}\left(r^{1}, \ldots, r^{N}\right)$, $j=1,2, \ldots$, satisfy system (83) for every solution (43).

Using transformations (29), we can set

$$
F\left(p, u_{1}, \ldots, u_{n}\right)=p
$$

without restricting the generality.

A calculation analogous to the one at the end of Sec. 2 results in an infinite triangular GT system corresponding to the given integrable chain. 
Definition 10 We call a compatible system of the form

$$
\begin{aligned}
& \partial_{i} p_{j}=f\left(p_{i}, p_{j}, u_{1}, \ldots, u_{n}\right) \partial_{i} u_{1}, \\
& \partial_{i} u_{k}=g_{k}\left(p_{i}, u_{1}, \ldots, u_{k}\right) \partial_{i} u_{1}, \quad k=1,2, \ldots, \\
& \partial_{i} \partial_{j} u_{1}=h\left(p_{i}, p_{j}, u_{1}, \ldots, u_{n}\right) \partial_{i} u_{1} \partial_{j} u_{1},
\end{aligned}
$$

where $i, j=1, \ldots, N, i \neq j$, a triangular $G T$ system. Here, $p_{1}, \ldots, p_{N}$ and $u_{1}, u_{2}, \ldots$ are functions of $r^{1}, \ldots, r^{N}$ and $\partial_{i}=\partial / \partial r^{i}$.

Substituting $u_{i}=u_{i}\left(r^{1}, \ldots, r^{N}\right), i=1,2, \ldots$, in the chain, calculating the derivatives with respect to $t$ and $x$ by virtue of (43), and equating the coefficients of $r_{x}^{s}$ to zero, we obtain

$$
\partial_{s} u_{\alpha} p_{s}=\phi_{\alpha, 1} \partial_{s} u_{1}+\cdots+\phi_{\alpha, \alpha+1} \partial_{s} u_{\alpha+1}, \quad \alpha=1,2, \ldots
$$

Using (87) and replacing $p_{s}$ with $p$, we find

$p=\phi_{1,1}+\phi_{1,2} g_{2}, \quad p g_{2}=\phi_{2,1}+\phi_{2,2} g_{2}+\phi_{2,3} g_{3}, \quad p g_{3}=\phi_{3,1}+\phi_{3,2} g_{2}+\phi_{3,3} g_{3}+\phi_{3,4} g_{4}$,

Solving this system for $g_{2}, g_{3}, \ldots$, we obtain

$$
g_{i}(p)=\psi_{i, 0}+\psi_{i, 1} p+\cdots+\psi_{i, i-1} p^{i-1},
$$

where $\psi_{i, j}$ are some functions of $u_{1}, \ldots, u_{i}$. In particular,

$$
g_{2}=-\frac{p}{\phi_{1,2}}-\frac{\phi_{1,1}}{\phi_{1,2}}
$$

Example 11a (continuation of Example 11). The triangular GT system corresponding to the Benney chain is

$$
\begin{aligned}
& \partial_{i} p_{j}=\frac{\partial_{i} u_{1}}{p_{i}-p_{j}}, \quad \partial_{i} \partial_{j} u_{1}=\frac{2 \partial_{i} u_{1} \partial_{j} u_{1}}{\left(p_{i}-p_{j}\right)^{2}} \\
& \partial_{i} u_{m}=\left(-(m-2) u_{m-2}-\cdots-2 u_{2} p_{i}^{m-2}-u_{1} p_{i}^{m-3}+p_{i}^{m-1}\right) \partial_{i} u_{1} .
\end{aligned}
$$

Equations (89) were first obtained in [12].

The compatibility conditions $\partial_{i} \partial_{j} u_{\alpha}=\partial_{j} \partial_{i} u_{\alpha}, \alpha=2,3,4$, result in a system of linear equations for $\partial_{i} p_{j}, \partial_{j} p_{i}$, and $\partial_{i} \partial_{j} u_{1}$. Solving it, we obtain

$$
\begin{aligned}
& \partial_{i} p_{j}=\frac{P\left(p_{i}, p_{j}\right)}{p_{i}-p_{j}} \partial_{i} u_{1}, \quad i \neq j, \\
& \partial_{i} \partial_{j} u_{1}=\frac{Q\left(p_{i}, p_{j}\right)}{\left(p_{i}-p_{j}\right)^{2}} \partial_{i} u_{1} \partial_{j} u_{1}, \quad i \neq j,
\end{aligned}
$$

where $P$ and $Q$ are quadratic polynomials in each of the variables $p_{i}$ and $p_{j}$. Their coefficients may in principle depend on $u_{1}, u_{2}, u_{3}$, and $u_{4}$. But it is easy 
to deduce from the compatibility conditions $\partial_{i} \partial_{j} p_{k}=\partial_{j} \partial_{i} p_{k}$ that $P$ and $Q$ depend only on $u_{1}$ and $u_{2}$.

We write (91) in the form

$$
\partial_{i} p_{j}=\left(\frac{R\left(p_{j}\right)}{p_{i}-p_{j}}+\left(z_{4} p_{j}^{2}+z_{5} p_{j}+z_{6}\right) p_{i}+z_{4} p_{j}^{3}+z_{3} p_{j}^{2}+z_{7} p_{j}+z_{8}\right) \partial_{i} u_{1}
$$

where $R(x)=z_{4} x^{4}+z_{3} x^{3}+z_{2} x^{2}+z_{1} x+z_{0}$. From the GT-system compatibility conditions, we easily obtain

$\partial_{i} \partial_{j} u_{1}=\left(\frac{2 z_{4} p_{i}^{2} p_{j}^{2}+z_{3} p_{i} p_{j}\left(p_{i}+p_{j}\right)+z_{2}\left(p_{i}^{2}+p_{j}^{2}\right)+z_{1}\left(p_{i}+p_{j}\right)+2 z_{0}}{\left(p_{i}-p_{j}\right)^{2}}+z_{9}\right) \partial_{i} u_{1} \partial_{j} u_{1}$.

Using change of variables (84), we can set the coefficient $z_{9}$ equal to $z_{6}-z_{7}$. The coefficients $z_{i}(x, y), i=1, \ldots, 8$, satisfy a pair of compatible dynamical systems in $y$ and $x$. The first of these systems is

$$
\begin{array}{lll}
z_{0, y}=2 z_{0} z_{5}-z_{1} z_{6}, & z_{1, y}=4 z_{0} z_{4}+z_{1} z_{5}-2 z_{2} z_{6}, \\
z_{2, y}=3 z_{1} z_{4}-3 z_{3} z_{6}, & z_{3, y}=2 z_{2} z_{4}-z_{3} z_{5}-4 z_{4} z_{6}, \\
z_{4, y}=z_{3} z_{4}-2 z_{4} z_{5}, & z_{5, y}=z_{4} z_{7}-z_{4} z_{6}-z_{5}^{2}, \\
z_{6, y}=z_{4} z_{8}-z_{5} z_{6}, & z_{7, y}=2 z_{1} z_{4}-2 z_{3} z_{6}-z_{5} z_{6}+z_{4} z_{8}, \\
z_{8, y}=2 z_{0} z_{4}-z_{6}^{2}-z_{6} z_{7}+z_{5} z_{8} . &
\end{array}
$$

The second of these systems looks more complicated. To linearize these systems, we reduce the polynomial $R$ to a canonical form, sacrificing normalization (86) for this.

It turns out that if the transformation coefficients

$$
p_{i}=\frac{a \bar{p}_{i}+b}{\bar{p}_{i}-\psi}, \quad i=1, \ldots, N,
$$

satisfy the conditions

$$
a_{u_{2}}=z_{4}(b+a \psi), \quad b_{u_{2}}=z_{4} b \psi+z_{5} b-z_{6} a, \quad \psi_{u_{2}}=z_{4} \psi^{2}+z_{5} \psi+z_{6},
$$

then the transformation preserves the form of Eqs. (93) and (94). The polynomial $R$ changes simply under transformations (95):

$$
R\left(p_{i}\right) \rightarrow\left(p_{i}-\psi\right)^{4} R\left(\frac{a p_{i}+b}{p_{i}-\psi}\right) .
$$

We first assume that all the roots of $R$ are distinct. We can then verify that using admissible transformations (95), we can set three of the four roots to be 0,1 , and $\infty$. The GT-system compatibility conditions then state that the fourth root $\lambda\left(u_{1}, u_{2}\right)$ is independent of $u_{2}$. Using transformations of the form $u_{1} \rightarrow q\left(u_{1}\right)$, 
we obtain either $\lambda=u_{1}$ or $\lambda=$ const. The GT-system compatibility conditions then imply that in the first case, Eqs. (93) and (94) coincide with the formulas in Example 2, where $P(x)=x(x-1)$, while the second case cannot be realized.

In the case of multiple roots, we can reduce the polynomial $R(x)$ to one of the canonical forms $R=0, R=1, R=x, R=x^{2}$, or $R=x(x-1)$. In all these cases, Eqs. (93) and (94) coincide with the corresponding equations in Example 1.

Below, we consider the general position case $R(x)=x(x-1)\left(x-u_{1}\right)$ and the most degenerate case $R(x)=0$.

Because we use transformations (95) when reducing $R$ to a canonical form, the functions $g_{k}$ in system (87) change their form from polynomial to rational functions with the denominator $(p-\psi)^{k-1}$. To construct them, we must describe all possible fractional-rational functions $g_{2}$. Using a transformation of the form $\bar{u}_{2}=\sigma\left(u_{1}, u_{2}\right)$ in the general position case, we can bring every such function $g_{2}$ to one of the functions

$$
\begin{aligned}
& \text { 1. } g_{2}(p)=\frac{u_{2}\left(u_{2}-1\right)\left(p-u_{1}\right)}{u_{1}\left(u_{1}-1\right)\left(p-u_{2}\right)} \quad \text { (the regular extension), } \\
& \text { 2. } g_{2}(p)=\frac{1}{p-u_{1}}, \\
& \text { 3. } g_{2}(p)=\frac{u_{1}^{-\lambda}\left(u_{1}-1\right)^{\lambda-1}}{p-\lambda}, \quad \lambda=1,0, \\
& \text { 4. } g_{2}(p)=\frac{u_{1}-u_{2}}{u_{1}\left(u_{1}-1\right)} p+\frac{u_{2}-1}{u_{1}-1} .
\end{aligned}
$$

The regular extension, case 1, is invariant under the discrete automorphisms described in Example 2. The other three cases are equivalent, and we can consider case 4 as an example.

We now consider the general position case 1 . The next step in the classification is finding fractional-rational GT families with the coefficients depending on $u_{1}$ and $u_{2}$. Such a general form family is determined by formulas (50) and (51), where $n=2$ and $k=1$. But we have the additional constraint that a zero of the denominator must coincide with a zero of the numerator of $g_{2}$, i.e., it must be equal $u_{2}$. It is easy to verify that this condition is equivalent to $s_{2}=0$ and $h_{1, u_{2}}=h_{2, u_{2}}=0$ in system (47), (48). The last condition implies that $h_{1}\left(u_{1}\right)$ and $h_{2}\left(u_{1}\right)$ are linearly independent solutions of the standard hypergeometric equation

$$
u(u-1) h(u)^{\prime \prime}+\left[s_{1}+s_{3}-\left(s_{3}+s_{4}+2 s_{1}\right) u\right] h(u)^{\prime}+s_{1}\left(s_{1}+s_{3}+s_{4}+1\right) h(u)=0 .
$$

Without restricting the generality, we can choose

$$
h_{3}\left(u_{1}, u_{2}\right)=\int_{0}^{u_{2}}\left(t-u_{1}\right)^{s_{1}} t^{s_{3}}(t-1)^{s_{4}} d t .
$$


As a result, we obtain

$$
F\left(p, u_{1}, u_{2}\right)=\frac{f_{1}\left(u_{1}, u_{2}\right) p-f_{2}\left(u_{1}, u_{2}\right)}{p-u_{2}},
$$

where

$$
\begin{aligned}
& f_{1}=\frac{u_{2}\left(u_{2}-1\right) h_{1} h_{3, u_{2}}+u_{1}\left(u_{1}-1\right)\left(h_{1} h_{3, u_{1}}-h_{3} h_{1}^{\prime}\right)}{u_{1}\left(u_{1}-1\right)\left(h_{1} h_{2}^{\prime}-h_{2} h_{1}^{\prime}\right)}, \\
& f_{2}=\frac{u_{1} u_{2}\left(u_{2}-1\right) h_{1} h_{3, u_{2}}+u_{2} u_{1}\left(u_{1}-1\right)\left(h_{1} h_{3, u_{1}}-h_{3} h_{1}^{\prime}\right)}{u_{1}\left(u_{1}-1\right)\left(h_{1} h_{2}^{\prime}-h_{2} h_{1}^{\prime}\right)} .
\end{aligned}
$$

We note that $h_{1} h_{2}^{\prime}-h_{2} h_{1}^{\prime}=$ const $\cdot\left(u_{1}-1\right)^{s_{1}+s_{4}} u_{1}^{s_{1}+s_{3}}$.

For special values of the parameters, we can solve the hypergeometric equation in elementary functions and find $F$ explicitly. For example, we have

$$
F=\frac{\left(u_{2}-u_{1}\right)^{s_{1}+1} u_{2}^{s_{3}+1}\left(u_{2}-1\right)^{-1-s_{1}-s_{3}}}{p-u_{2}},
$$

for $s_{4}=-2-s_{1}-s_{3}$ and

$$
F=\frac{(p-1)\left(u_{2}-u_{1}\right)^{s_{1}+1} u_{2}^{s_{3}+1}\left(u_{1}-1\right)^{-1-s_{1}}}{p-u_{2}}
$$

for $s_{4}=0$.

Further, we must find the functions $g_{3}, g_{4}, \ldots$ in system (87). These functions are defined up to an arbitrary transformation (84), where $\alpha=3,4, \ldots$. To fix them more or less rigidly, it is convenient to assume that the functions $g_{3}, g_{4}, \ldots$ are linear in $u_{i}, i>2$ (cf. (90)). In particular, we can choose

$$
\begin{aligned}
g_{3}(p)= & -\frac{\left(u_{1}-u_{2}\right)\left(u_{2}-1\right) p}{u_{1}\left(u_{1}-1\right)\left(p-u_{2}\right)^{2}}, \\
g_{i}(p)= & \frac{(i-3)\left(u_{1}-u_{2}\right)\left(u_{2}-1\right) p u_{i}}{u_{1}\left(u_{1}-1\right)\left(p-u_{2}\right)^{2}}-\frac{\left(u_{1}-u_{2}\right)^{i-3}\left(u_{2}-1\right)^{2} p\left(p-u_{1}\right)(p-1)^{i-4}}{u_{1}\left(u_{1}-1\right)^{i-2}\left(p-u_{2}\right)^{i-1}}- \\
& -\sum_{s=1}^{i-4} \frac{(i-s-2)\left(u_{1}-u_{2}\right)^{s}\left(u_{2}-1\right)^{2} p\left(p-u_{1}\right)(p-1)^{s-1} u_{i-s}}{u_{1}\left(u_{1}-1\right)^{s+1}\left(p-u_{2}\right)^{s+2}}, \quad i>3 .
\end{aligned}
$$

The coefficients $\phi_{i, j}$ of the corresponding chain (83) can be determined from the relations

$$
\begin{aligned}
& F(p)=\phi_{1,1}+\phi_{1,2} g_{2}, \quad F(p) g_{2}=\phi_{2,1}+\phi_{2,2} g_{2}+\phi_{2,3} g_{3}, \\
& F(p) g_{3}=\phi_{3,1}+\phi_{3,2} g_{2}+\phi_{3,3} g_{3}+\phi_{3,4} g_{4}, \quad \ldots,
\end{aligned}
$$


where $F$ is given by formula (97). These relations are equivalent to an infinite triangular system of linear algebraic equations. Solving it, we obtain

$$
\begin{array}{ll}
\phi_{1,1}=\frac{f_{1} u_{1}-f_{2}}{u_{1}-u_{2}}, & \phi_{1,2}=-\frac{u_{1}\left(u_{1}-1\right)\left(f_{1} u_{2}-f_{2}\right)}{u_{2}\left(u_{2}-1\right)\left(u_{1}-u_{2}\right)} \\
\phi_{2,1}=\frac{\left(u_{2}-1\right)\left(f_{1} u_{2}-f_{2}\right)}{\left(u_{1}-1\right)\left(u_{1}-u_{2}\right)}, & \phi_{2,2}=\frac{f_{2} u_{1}-f_{1} u_{2}^{2}}{u_{2}\left(u_{1}-u_{2}\right)} \\
\phi_{2,3}=f_{1} u_{2}-f_{2}, & \ldots
\end{array}
$$

All the functions $\phi_{i, j}, i>2$, are linear combinations of $f_{1}$ and $f_{2}$ with rational coefficients with the same denominator $\left(u_{2}-1\right)\left(u_{1}-u_{2}\right)$ and a numerator depending on $u_{1}, \ldots, u_{i+1}$.

We next consider the most degenerate case $R(x)=0$. In this case, the GT system is holomorphic on the diagonal. According to conjecture 1 (see Appendix (C), precisely the models related to such GT systems admit multidimensional generalizations.

We can easily verify that in the case $R(x)=0$, the triangular system is equivalent to the system

$$
\partial_{i} p_{j}=0, \quad \partial_{i} \partial_{j} u_{1}=0, \quad \partial_{i} u_{k}=p_{i}^{k-1} u_{1}, \quad k=2,3, \ldots
$$

Automorphisms of this system are generated by the transformations

$$
\begin{array}{ll}
p_{j} \rightarrow p_{j}, & u_{i} \rightarrow \nu u_{i}+\gamma_{i}, \\
p_{j} \rightarrow a p_{j}+b, & u_{i} \rightarrow a^{i-1} u_{i}+(i-1) a^{i-2} b u_{i-2}+\cdots+b^{i-1} u_{1},
\end{array}
$$

where $j=1, \ldots, N, i=1,2, \ldots$ The corresponding GT family is $F(p)=$ $A\left(u_{1}, u_{2}\right) p+B\left(u_{1}, u_{2}\right)$. The coefficients $A(x, y)$ and $B(x, y)$ are found from the semi-Hamiltonian condition, which is equivalent to the system of differential equations

$$
\begin{aligned}
& A B_{y y}=A_{y} B_{y}, \quad A B_{x y}=A_{y} B_{x}, \quad A B_{x x}=A_{x} B_{x}, \\
& A A_{y y}=A_{y}^{2}, \quad A A_{x y}=A_{x} A_{y}, \quad A A_{x x}=A_{x}^{2}+A_{x} B_{y}-A_{y} B_{x} .
\end{aligned}
$$

We can easily solve this system in elementary functions. For each solution of it, formulas (98) determine the corresponding integrable chain (83).

Formulas (101) imply that $F$ can depend on $u_{2}$ in two different ways:

1. $F\left(p, u_{1}, u_{2}\right)=e^{\lambda u_{2}}\left(a\left(u_{1}\right) p+b\left(u_{1}\right)\right)$ in the general position case, or

2. $F\left(p, u_{1}, u_{2}\right)=a\left(u_{1}\right) p+\lambda u_{2}+b\left(u_{1}\right)$.

In the first case, we in turn have two possibilities: $b^{\prime} \neq 0$ and $b^{\prime}=0$. We have $a=\sigma^{\prime}, \quad b=k_{1} \sigma, \quad \sigma(x)=c_{1} e^{\mu_{1} x}+c_{2} e^{\mu_{2} x}, \quad$ where $c_{1} c_{2}\left(\lambda k_{1}-\mu_{1} \mu_{2}\right)=0$, 
and

$$
b=c_{1}, \quad a(x)=c_{2} e^{\mu x}+c_{3}, \quad \text { where } c_{2}\left(c_{1} \lambda-c_{3} \mu\right)=0,
$$

for $b^{\prime}=0$. The same variants in case 2 correspondingly give

$$
a=\sigma^{\prime}, \quad b=k_{1} \sigma, \quad \sigma(x)=c_{1}+c_{2} x+c_{3} e^{\mu x}, \quad \text { where } c_{3}\left(\lambda-c_{2} \mu\right)=0,
$$

and

$$
b=c_{1}, \quad a(x)=c_{2} e^{\mu x}+c_{3}, \quad \text { where } c_{2}\left(\lambda-c_{3} \mu\right)=0 .
$$

It is easy to see that in the general position case, transformation (100) reduces the function $F$ to the form

$$
F(p)=e^{u_{2}+u_{1}}(p-1)+e^{u_{2}-u_{1}}(p+1) .
$$

The corresponding integrable chain is

$$
u_{k, t}=\left(e^{u_{2}+u_{1}}+e^{u_{2}-u_{1}}\right) u_{k+1, x}+\left(e^{u_{2}-u_{1}}-e^{u_{2}+u_{1}}\right) u_{k, x}, \quad k=1,2, \ldots .
$$

This chain has an infinite hierarchy of commuting flows as usual. For instance, the next flow is given by

$$
\begin{aligned}
u_{k, \tau}= & \left(e^{u_{2}+u_{1}}+e^{u_{2}-u_{1}}\right) u_{k+2, x}+\left(u_{3}-u_{1}\right)\left(e^{u_{2}+u_{1}}+e^{u_{2}-u_{1}}\right) u_{k+1, x}+ \\
& +\left(e^{u_{2}+u_{1}}\left(u_{1}-u_{3}-1\right)+e^{u_{2}-u_{1}}\left(u_{3}-u_{1}-1\right)\right) u_{k, x}, \quad k=1,2, \ldots
\end{aligned}
$$

In case 2 with $c_{3}=\lambda=0$ and $k_{1}=1$, we obtain the chain

$$
u_{k, t}=u_{k+1, x}+u_{1} u_{k, x}, \quad k=1,2, \ldots,
$$

which is equivalent to universal hierarchy chain 48. Chain (103) is a degeneration of the chain

$$
u_{k, t}=u_{k+1, x}+u_{2} u_{k, x}, \quad k=1,2, \ldots
$$

Following [15, 41, we can easily construct $(2+1)$-dimensional generalizations of the $(1+1)$-dimensional chains described above. Namely, some families of the obtained functions $F$ depend linearly on two parameters. We let $\gamma_{1}$ and $\gamma_{2}$ denote these parameters. The corresponding integrable chain

$$
u_{k, t}=\gamma_{1}\left(\phi_{k, 1} u_{1, x}+\cdots+\phi_{k, k+1} u_{k+1, x}\right)+\gamma_{2}\left(\psi_{k, 1} u_{1, x}+\cdots+\psi_{k, k+1} u_{k+1, x}\right)
$$

is also linear in $\gamma_{1}$ and $\gamma_{2}$. We claim that the $(2+1)$-dimensional chain

$$
u_{k, t}=\left(\phi_{k, 1} u_{1, x}+\cdots+\phi_{k, k+1} u_{k+1, x}\right)+\left(\psi_{k, 1} u_{1, y}+\cdots+\psi_{k, k+1} u_{k+1, y}\right)
$$

is integrable from the standpoint of the hydrodynamic reduction method. We can easily describe these reductions in each actual case. For example, we have

$$
F(p)=\gamma_{1} e^{u_{2}+u_{1}}(p-1)+\gamma_{2} e^{u_{2}-u_{1}}(p+1)
$$


in the general position case. The corresponding $(2+1)$-dimensional chain (105) is

$$
u_{k, t}=e^{u_{2}+u_{1}}\left(u_{k+1, x}-u_{k, x}\right)+e^{u_{2}-u_{1}}\left(u_{k+1, y}+u_{k, y}\right), \quad k=1,2, \ldots
$$

Its hydrodynamic reductions are given by

$$
r_{t}^{i}=\left(e^{u_{2}+u_{1}}\left(p_{i}-1\right)+e^{u_{2}-u_{1}} q_{i}\left(p_{i}+1\right)\right) r_{x}^{i}, \quad r_{y}^{i}=q_{i} r_{x}^{i},
$$

where

$$
\begin{aligned}
& \partial_{i} u_{k}=p_{i}^{k-1} u_{1}, \quad k=1,2, \ldots, \quad \partial_{i} \partial_{j} u_{1}=0, \quad \partial_{i} p_{j}=0, \\
& \partial_{i} q_{j}=\frac{\left(q_{i}-q_{j}\right)\left[\left(p_{i}+1\right)\left(p_{j}-1\right) e^{u_{1}}+q_{j}\left(p_{i}-1\right)\left(p_{j}+1\right) e^{-u_{1}}\right]}{\left(p_{i}-p_{j}\right)\left(e^{u_{1}}+q_{i} e^{-u_{1}}\right)} \partial u_{1} .
\end{aligned}
$$

Gibbons-Tsarev system (106) with two-dimensional fibers (see Appendix C) deserves a separate study.

We now consider one of the degenerate cases $F=\gamma_{1} e^{u_{1}} p+\gamma_{2}\left(p+u_{2}\right)$. The corresponding (2+1)-dimensional integrable generalization of chain (104) is

$$
u_{k, t}=e^{u_{1}} u_{k+1, x}+u_{k+1, y}+u_{2} u_{k, y}, \quad k=1,2, \ldots
$$

The hydrodynamic reductions are described by

$$
r_{t}^{i}=\left[e^{u_{1}} p_{i}+q_{i}\left(p_{i}+u_{2}\right)\right] r_{x}^{i}, \quad r_{y}^{i}=q_{i} r_{x}^{i},
$$

where

$$
\begin{aligned}
& \partial_{i} u_{k}=p_{i}^{k-1} u_{1}, \quad k=1,2, \ldots, \quad \partial_{i} \partial_{j} u_{1}=0, \quad \partial_{i} p_{j}=0, \\
& \partial_{i} q_{j}=\frac{\left(q_{i}-q_{j}\right)\left(p_{i} q_{j}+e^{u_{1}} p_{j}\right)}{\left(p_{i}-p_{j}\right)\left(q_{i}+e^{u_{1}}\right)} \partial u_{1} .
\end{aligned}
$$

We note that the GT system for this $(2+1)$-dimensional chain is equivalent to GT system (106).

Conjecture 2 Every integrable chain (105) can be generated by a two-dimensional linear family of solutions of system (101) using the construction described above.

\section{E List of unsolved problems}

Here, we formulate several unsolved problems that from our standpoint seem important for better understanding the nature of the GT system.

Problem 1 To find all solutions of system (32) of functional equations up to an equivalence.

Problem 2 To describe all two-field extensions of system (33).

Problem 3 To describe all solutions of Eq. (25) for GT system (45), (46) (at least for small $n$ ).

Problem 4 To construct solutions of Eq. (25) and the pseudopotential in the case of the regular GT-system extension in Example 4. 


\section{Acknowledgments}

The authors are grateful to E. V. Ferapontov, who several years ago drew their attention to interesting unsolved problems in the framework of the hydrodynamic reduction method. The authors thank F. F. Voronov, B. A. Dubrovin, I. M. Krichever, O. I. Mokhov, M. V. Pavlov, V. P. Spiridonov, B. L. Feigin, E. V. Ferapontov, and A. B. Shabat for the useful discussions. One of the authors (V. V. S.) is grateful to Brock University for the hospitality.

This work was supported in part by the Russian Foundation for Basic Research (Grant Nos. 08-01-464?? and 09-01-92442) and by the Program for Supporting Leading Scientific Schools (Grant No. NSh-3472.2008.2).

\section{References}

[1] Alan C. Newell, Solitons in Mathematics and Physics, CBMS-NSF Reg. Conf. Ser. Appl. Math., VOl. 48, SIAM, Philadelphia, Pa., 1985

[2] L. A. Takhtadzhyan and L. D. Faddeev, Hamiltonian Approach to the Theory of Solitons, Moscow, Nauka, 1986, in Russian; English transl.: L. D. Faddeev and L. A. Takhtadzhyan, The Hamiltonian Methods in the Theory of Solitons, Springer, Berlin, 1987

[3] V. E. Zakharov, ed. What is Integrability? Springer, Berlin, 1991

[4] A. V. Mikhailov, Integrability, Lect. Notes Phys., Vol. 767, Berlin, Springer, 2009

[5] E. V. Zakharov, Dispersionless limit of integrable systems in $2+1$ dimensions, Singular Limits of Dispersive Waves, NATO ASI Ser. B Phys., Vol. 320, N. M. Ercolani, I. R. Gabitov, C. D. Levermore, and D. Serre, eds. Plenum, New York, 1994, 165-174

[6] I. M. Krichever, Comm. Pure Appl. Math., 47, 1994, 437-475

[7] A. V. Odesskii and V. V. Sokolov, Funct. Anal. Appl., 2008, 42, 3, 205-212

[8] V. E. Zakharov and A. B. Shabat, Funct. Anal. Appl., 1979, 13, 3, 166-174

[9] M. V. Saveliev and A. M. Vershik, Comm. Math. Phys., 126, 1989, 367-378

[10] S. V. Manakov and P. M. Santini, Phys. Lett. A, 359, 2006, 613-619

[11] S. V. Manakov and P. M. Santini, Theor. Math. Phys., 152, 2007, 10041011

[12] J. Gibbons and S. P. Tsarev, Phys. Lett. A, 211, 1996, 19-24

[13] E. V. Ferapontov and K. R. Khusnutdinova, Comm. Math. Phys., 248, 2004, 187-206 
[14] E. V. Ferapontov and K. R. Khusnutdinova, J. Phys. A, 37, 2004, 29492963

[15] E. V. Ferapontov and K. R. Khusnutdinova, J. Math. Phys., 45, 2004, $2365-2377$

[16] A. V. Odesskii, Selecta Math., 13, 2008, 727-742, arXiv:0704.3577v3 [math.AP], 2007

[17] E. V. Ferapontov, K. R. Khusnutdinova, and S. P. Tsarev, Comm. Math. Phys., 261, 2006, 225-243

[18] E. V. Ferapontov and A. V. Odesskii, Integrable Lagrangians and modular forms, arXiv:0707.3433v2 [nlin.SI], 2007

[19] P. A. Burovskiy, E. V. Ferapontov, and S. P. Tsarev, Second order quasilinear PDEs and conformal structures in projective space, arXiv:0802.2626v 3 [nlin.SI], 2008

[20] A. V. Odesskii and V. V. Sokolov, Integrable pseudopotentials related to generalized hypergeometric functions, arXiv:0803.0086v3 [nlin.SI], 2008

[21] I. M. Gel'fand, M. I. Graev, and V. S. Retakh, Russ. Math. Surveys, 47, 1992, 1-88

[22] E. V. Ferapontov, L. Hadjikos, and K. R. Khusnutdinova, Integrable equations of the dispersionless Hirota type and hypersurfaces in the Lagrangian Grassmannian, arXiv:0705.1774v1 [math.DG], 2007

[23] M. V. Pavlov, Theor. Math. Phys., 138, 2004, 45-58

[24] M. V. Pavlov, J. Phys. A, 39, 2006, 10803-10819

[25] E. V. Ferapontov and D. G. Marshal, Math. Ann., 339, 2007, 61-99

[26] I. M. Krichever, Comm. Math. Phys., 143, 1992, 415-429

[27] A. V. Odesskii, M. V. Pavlov, and V. V. Sokolov, Theor. Math. Phys., 154, 2008, 209-219, arXiv:0710.5655v2 [nlin.SI], 2007

[28] A. V. Odesskii and V. V. Sokolov, Theor. Math. Phys., 2009, 161, 13401352 arXiv:0810.3879v1 [nlin.SI] 2008

[29] V. P. Spiridonov, Russ. Math. Surveys, 2008, 63, 405-472

[30] K. Löwner, Math. Ann., 89, 1923, 103-121

[31] K. Takasaki and T. Takebe, Rev. Math. Phys., 7, 1995, 743-808

[32] V. Shramchenko, J. Phys. A, 36, 2003, 10585-10605

[33] T. Takebe, L.-P. Leo, and A. Zabrodin, J. Phys. A, 39, 2006, 11479-11501 
[34] M. Mañas, E. Medina, and L. Martínes Alonso, J. Phys. A, 39, 2006, 23492381

[35] A. V. Mikhailov and V. V. Sokolov, Symmetries of differential equations and the problem of integrability, Integrability, Lect. Notes Phys., Vol. 767, A. V. Mikhailov, ed. 767, Berlin, Springer, 2009, 19-88

[36] A. V. Mikhailov and R. I. Yamilov, J. Phys. A, 31, 1998, 6707-6715

[37] B. A. Dubrovin and S. P. Novikov, Russ. Math. Surveys, 1989, 44, 35-124

[38] S. P. Tsarëv, Math. USSR-Izv., 1991, 37, 397-419

[39] S. P. Tsarëv, Sov. Math. Dokl., 31, 1985, 488-491

[40] E. V. Ferapontov, Phys. Lett. A, 158, 1991, 112-118

[41] M. V. Pavlov, J. Math. Phys., 44, 2003, 4134-4156

[42] M. V. Pavlov, Comm. Math. Phys., 272, 2007, 469-505

[43] E. V. Ferapontov, K. R. Khusnutinova, and C. Klein, On linear degeneracy of integrable quasilinear systems in higher dimensions, arXiv:0909.5685v2 [nlin.SI], 2009

[44] A. V. Odesskii and V. V. Sokolov, Systems of Gibbons-Tsarev type and integrable 3-dimensional models, arXiv:0906.3509v1 [nlin.SI], 2009

[45] D. J. Benney, Stud. Appl. Math., 52, 1973, 45-50

[46] B. A. Kupershmidt and Yu. I. Manin, Funct. Anal. Appl., 11, 3, 1977, 188-197

[47] V. E. Zakharov, Phys. D, 3, 1981, 193-202

[48] L. Martínez Alonso and A. B. Shabat, Theor. Math. Phys., 140, 2004, 1073-1085 